\title{
Order-disorder molecular model of the smectic- $A$-smectic- $C$ phase transition in materials with conventional and anomalously weak layer contraction
}

\author{
M. V. Gorkunov, ${ }^{1,2}$ M. A. Osipov, ${ }^{1}$ J. P. F. Lagerwall, ${ }^{3}$ and F. Giesselmann ${ }^{3}$ \\ ${ }^{1}$ Department of Mathematics, University of Strathclyde, Glasgow G1 1XH, United Kingdom \\ ${ }^{2}$ Institute of Crystallography, Russian Academy of Sciences, 119333 Moscow, Russia \\ ${ }^{3}$ Institute of Physical Chemistry, University of Stuttgart, 70569 Stuttgart, Germany
}

(Received 17 July 2007; published 19 November 2007)

\begin{abstract}
We develop a molecular-statistical theory of the smectic- $A$-smectic- $C$ transition which is described as a transition of the order-disorder type. The theory is based on a general expansion of the effective interaction potential and employs a complete set of orientational order parameters. All the order parameters of the smectic$C$ phase including the tilt angle are calculated numerically as functions of temperature for a number of systems which correspond to different transition scenario. The effective interaction potential and the parameters of the transition are also calculated for specific molecular models based on electrostatic and induction interaction between molecular dipoles. The theory successfully reproduces the main properties of both conventional and so-called "de Vries-type" smectic liquid crystals, clarifies the origin of the anomalously weak layer contraction and describes the tricritical behavior at the smectic- $A$-smectic- $C$ transition. The "de Vries behavior," i.e., anomalously weak layer contraction is also obtained for a particular molecular model based on interaction between longitudinal molecular dipoles. A simple phenomenological model is presented enabling one to obtain explicit expressions for the layer spacing and the tilt angle which are used to fit the experimental data for a number of materials.
\end{abstract}

DOI: 10.1103/PhysRevE.76.051706

PACS number(s): 64.70.Md, 77.80.Bh, 42.70.Df

\section{INTRODUCTION}

Smectic liquid crystals form layered phases with orientational ordering of anisotropic molecules. In the simplest smectic- $A(\operatorname{Sm} A)$ phase, the long molecular axes are on average parallel to the smectic layer normal and the phase is uniaxial. In contrast, in the smectic- $C(\mathrm{SmC})$ phase the molecules are inclined on average at an angle $\Theta$ with respect to the layer normal. Tilted smectic phases attract a significant attention mainly because of the rich variety of such phases with unusual three dimensional structure [1,2], which also possess ferroelectric, antiferroelectric, and ferrielectric properties. On the other hand, ferro- and antiferroelectric tilted smectic materials are extremely promising for the new generation of fast electro-optic displays as well as various nondisplay applications [3]. One notes also that tilted phases of exactly the same point symmetry as the thermotropic $\mathrm{SmC}$ phase exist also in other orientationally ordered soft systems including, in particular, mesogenic polymers, lamellar $L_{\beta}$ phase and smectic- $C$ elastomers, which receive increasing attention [4-6].

The molecular theory of the $\operatorname{Sm} A-\operatorname{Sm} C$ phase transition and the microscopic origin of the tilt in the $\mathrm{SmC}$ phase have been an issue of debate ever since the first observation of the temperature-dependent tilt angle $\Theta$ in early 1970s [7]. A number of molecular models have been proposed [8-16] based on different intermolecular interactions and packing effects. For example, it is well known that the molecules exhibiting the $\mathrm{SmC}$ phase usually possess transverse dipoles. Thus McMillan [8] showed that a rotational freezing of such molecules could result in a tilt. A similar model was proposed by Wulf [9] who considered a model interaction between zig-zag shaped molecules.

One notes, however, that these models, as well as the steric model proposed by Somoza and Tarazona [10], empha- size the ordering of short molecular axes as a necessary condition for the tilt. On the other hand, the tilt may occur due to interactions between uniaxial molecules, and one may expect this mechanism to be predominant in most systems due to the strength of the coupling between long axes of strongly anisotropic mesogenic molecules. Among the "uniaxial" models the most successful one was proposed by van der Meer and Vertogen [11] who considered an induced interaction between the permanent dipole and a polarizability of freely rotating adjacent molecules. However, as shown by Goossens [12], this model describes a change of structure rather than a realistic order-disorder phase transition. Goossens himself pointed out that biaxial molecular quadrupoles could give rise to a tilt of the director but he did not obtain any general results. Nevertheless, Poniewierski and Sluckin [13] have shown that the tilt in the smectic-C phase can be stabilized by an interaction between uniaxial quadrupoles, the important case which is in contradiction with the Goossens model, and which emphasizes the importance of interactions between long molecular axes.

The significance of uniaxial intermolecular interactions is also supported by recent computer simulations of the smectic- $C$ phase $[14,15]$. It has been shown that the $\mathrm{Sm} A-\mathrm{Sm} C$ transition can indeed be induced by electrostatic interaction between uniaxial molecular quadrupoles [14] or by interaction between pairs of antiparallel longitudinal dipoles [15]. In contrast, transverse molecular dipoles appear to be much less important. Finally Govind and Madhusudana [16] have recently presented a model based on electrostatic interaction between off-axes transverse molecular dipoles, which does not average out to zero by a rotation about the long molecular axis. However, despite all these efforts, there is still no satisfactory realistic molecular theory of the $\mathrm{SmC}$ phase. In particular, none of the existing theories can de- 
scribe the first order smectic- $A-$ smectic- $C$ transition which is often observed experimentally [1]. The existing models also cannot be used to describe the properties of novel smectic liquid crystals with weak layer contraction.

When a liquid crystal undergoes a transition from the $\operatorname{Sm} A$ phase to the $\mathrm{SmC}$ phase, in most materials the layer spacing $p$ decreases by a factor of $\cos \Theta[3,17,18]$. This layer shrinkage appears to be a very negative factor in manufacturing and operation of electro-optic devices based on ferroand antiferroelectric smectic- $C^{*}$ liquid crystals. The main obstacle in commercialization of such devices is related to the problems determined by the contraction of smectic layers at the transition from the paraelectric $\mathrm{Sm} A^{*}$ phase to the ferroelectric $\mathrm{SmC}^{*}$ phase $[3,18,19]$. This contraction, together with a positional anchoring of smectic layers at the surfaces of a thin cell leads to a buckling of layers in a chevron geometry. The development of such chevron structures is accompanied by the formation of the so-called "zigzag" defects and a reduction in effective optical tilt angle. This seriously degrades the quality of electro-optic devices.

In fact, different materials show slightly varying degree of contraction across the $\mathrm{Sm} A-\mathrm{Sm} C$ phase transition. However, a surprise was the discovery that in some smectic liquid crystals there is almost no layer contraction at the tilting phase transition [20,21]. Recent works have demonstrated that there exist a number of smectic- $C^{*}$ materials with different molecular structure [20-23] displaying virtually constant smectic layer spacing. An understanding of why such materials do not show a contraction of the smectic layers at the $\mathrm{Sm} A^{*}-\mathrm{Sm} C^{*}$ transition, and why others do, is not only a key issue for the development of ferro- and antiferroelectric liquid crystal devices, but is also extremely interesting from a fundamental research point of view, and may modify our general concept of the whole class of tilting transitions in various soft matter systems.

In most models, either the positional and orientational orders are assumed to be ideal, or those two types of ordering are decoupled [24]. In either case the biaxiality of the molecular distribution in the $\mathrm{SmC}$ phase is neglected. Thus these models all adopt a conventional view of the transition in which it is assumed that molecular axes simply tilt away from the layer normal. In this picture, the $\operatorname{Sm} A-\operatorname{Sm} C$ phase transition has to be accompanied by substantial layer contraction determined by the factor of $\cos \Theta$.

An important exception is represented by a series of papers by de Vries [25-29] and Leadbetter [30] who developed a qualitative model which is known as the de Vries model. The model is based on the recognition that in all real smectic liquid crystals the orientational ordering of long molecular axes is not perfect. As a result, the mean square of the molecular tilt appears to be nonzero even in the $\operatorname{Sm} A$ phase and thus the most probable orientation of the long molecular axes is not along the layer normal but on the surface of the cone around the layer normal. The absence of a macroscopic optical tilt in the $\operatorname{Sm} A$ phase is then explained by a uniform distribution of the tilt directions (molecular azimuthal angles) in the layer plane. The transition to a tilted phase can occur in this model simply through an ordering of the azimuthal angles leading to a well defined macroscopic tilt direction. This ordering would not by itself produce any change in the layer spacing.
From the point of view of theoretical physics, the de Vries model corresponds to a different type of the tilting phase transition. In the conventional model, the $\operatorname{Sm} A-\operatorname{Sm} C$ transition is determined by the collective tilt of long molecular axes. Such a transition is not accompanied by the onset of any new ordering, and thus resembles a structural change. But in the de Vries model the transition is determined by the ordering of molecular azimuthal orientations and the director tilt occurs as a consequence. At the same time, the distribution of molecular polar angles (i.e., the angles between molecular axes and the layer normal), which determines the average layer spacing, remains basically the same.

De Vries model is inconsistent with high orientational order in the smectic- $C$ phase and recent observations support this. According to the results of $\mathrm{x}$-ray experiments $[31,32]$, the orientational order parameter in the $\mathrm{SmC}$ materials without layer contraction is substantially smaller than in conventional smectic liquid crystals. This conclusion is also approved by the order parameter measurements of a dichroic dye dissolved in the smectic- $C$ phase [32] and by recent IR spectroscopy measurements [33]. Moreover, experimental data indicate that the averaged orientational distribution of long molecular axes about the smectic layer normal is essentially the same in the $\operatorname{Sm} A$ and $\operatorname{Sm} C$ phases [31]. In contrast, the positional (smectic) order seems to be very high. This is compatible with the molecular structure of novel smectic materials containing either fluorinated chains or siloxane groups. These groups enhance layering due to partial microphase separation between different molecular fragments. The importance of this separation has also been revealed by recent experimental studies. In real smectics $C$ both mechanisms could in principle contribute. Indeed, recent $x$-ray and ellipsometric results indicate the existence of crossover behavior between conventional and "de Vries-type" smectics $A$ [34].

The primary goal of the present paper is to develop a molecular-statistical theory of the $\operatorname{Sm} A-\operatorname{Sm} C$ tilting transition in the presence of some orientational disorder. It is shown that such a model can be used to describe both conventional smectics and smectics with anomalously weak layer contraction. Moreover, it allows to trace how the details of the model interaction potential affect the scenario of the $\mathrm{Sm} A-\mathrm{Sm} C$ transition. Since the nematic order is not assumed to be high in this approach, the transition is described as a true order-disorder phase transition with complete set of orientational order parameters of the smectic- $C$ phase, which has been achieved in none of the previous models. Some preliminary results of this study have already been published [35].

The paper is arranged as follows. In Sec. II we present a simple phenomenological model of the $\operatorname{Sm} A-\operatorname{Sm} C$ transition which enables one to obtain explicit expressions for the tilt angle and smectic layer spacing, and to describe a crossover between the conventional and de Vries-type behavior. The expression for the layer spacing is used to fit the experimental data for a number of conventional and de Vries materials. Section III is devoted to the application of the density functional theory to smectic liquid crystal with perfect translational order and derivation of the order parameters. In that section temperature variation of all the order parameters is 
calculated numerically. Weak layer contraction and tricritical properties are considered in Secs. III C and III D, respectively. Section IV describes how the constants of the mean field potential can be related to particular model pair interactions of molecules with elongated core, dipole moments, and polarizability. Finally, we present the summary and draw the general conclusions in Sec. V.

\section{SIMPLE PHENOMENOLOGICAL MODEL}

Before developing a molecular statistical theory of the $\mathrm{Sm} A-\mathrm{SmC}$ transition let us first consider a simple phenomenological model which enables one to obtain explicit expressions for the layer spacing $p$ and the tilt angle $\Theta$ of the smectic- $C$ phase. One notes that the first model of this kind has been proposed by Chen and Lubensky long ago [36]. In this model, the free energy is considered as a function of the components of the wave vector $\mathbf{k}$ of the smectic density wave. In the smectic- $A$ phase, the wave vector $\mathbf{k}$ is parallel to the director $\mathbf{n}$ which is assumed to be along the $z$ axis. Then the transition into the smectic- $C$ phase is indicated by the appearance of the perpendicular to $z$ component $\mathbf{k}_{\perp}$ in the direction of the tilt.

As a generalization of this idea one may consider an expansion of the free energy of the smectic- $C$ phase in terms of both wave vector $\mathbf{k}$ and the orientational tensor order parameter $Q_{i j}=\left\langle a_{i} a_{j}-\delta_{i j} / 3\right\rangle$, where $\mathbf{a}$ is the unit vector of long molecular axis. Neglecting the small biaxiality of the smectic- $C$ phase the tensor order parameter can be expressed as $\mathbf{Q} \approx S(\mathbf{n} \cdot \mathbf{n}-\mathbf{I} / 3)$ where $S$ is the nematic scalar order parameter. Similar models based on such an expansion have been considered before $[37,38]$, but they have not been used to describe the variation of the smectic layer spacing. Now one can also include the experimental observation that the nematic order parameter $S$ is abnormally small in de Vries materials [1]. The free energy can then be expanded in powers of $\mathbf{Q}$, retaining for simplicity only linear and quadratic terms. As a result one obtains the following model free energy of the smectic- $C$ phase:

$$
\begin{aligned}
F_{C}= & F_{0}(S)-b_{1} S^{2} k^{2}-e_{1}(\mathbf{k} \cdot \mathbf{Q} \cdot \mathbf{k})+g_{1}(\mathbf{k} \cdot \mathbf{Q} \cdot \mathbf{Q} \cdot \mathbf{k}) \\
& +b_{2} S^{2} k^{4}+e_{2}(\mathbf{k} \cdot \mathbf{Q} \cdot \mathbf{k})^{2}+g_{2} k^{2}(\mathbf{k} \cdot \mathbf{Q} \cdot \mathbf{k}) \\
& +c k^{2}(\mathbf{k} \cdot \mathbf{Q} \cdot \mathbf{Q} \cdot \mathbf{k}) .
\end{aligned}
$$

Substituting the expression for $\mathbf{Q}$, we can rewrite the free energy in terms of $k_{z}=(\mathbf{k} \cdot \mathbf{n}), k^{2}=k_{z}^{2}+k_{\perp}^{2}$ and $S$. In particular, simple explicit expressions for $k$ and $\Theta$ can be obtained when $g_{1}=g_{2}=0$. In this case the free energy (1) can be rewritten in the following dimensionless form:

$$
\widetilde{F}_{C}=F(S)+\frac{1}{2}\left(q^{2}-1\right)^{2}+f_{1} S^{-1} \Delta+f_{2} \Delta^{2}+c^{\prime} k^{2} \Delta,
$$

where $\Delta=2 q^{2}-3 q_{\perp}^{2}$ and the dimensionless wave vector $\mathbf{q}$ $=\mathbf{k} / k_{0}$ with $k_{0}^{2}=\left(2 b_{2}^{\prime} / b_{1}\right)$ have been introduced. The dimensionless coefficients $f_{1}, f_{2}$, and $c^{\prime}$ are expressed as $f_{1}$ $=2 e_{1} / 3 b_{1}, f_{2}=2 e_{2} / 9 b_{2}^{\prime}, c^{\prime}=c / 9 b_{1}$, where $b_{2}^{\prime}=b_{2}-4 c / 27$, and the dimensionless free energy $\widetilde{F}_{C}=F_{C} 2 b_{2}^{\prime} / b_{1}^{2}$. Minimization of the free energy (2) yields $q^{2}=1-c^{\prime} \Delta$ and $\Delta=-\left(f_{1} S^{-1}\right.$ $\left.-c^{\prime}\right) / 2\left[f_{2}-\left(c^{\prime}\right)^{2}\right]$. Using these results one readily obtains the following explicit expressions for $k$ and $\Theta$ :

$$
\begin{gathered}
k_{C}^{2}=k_{0}^{2}\left(2 f_{2}-c^{\prime} f_{1} S^{-1}\right)\left[2 f_{2}-\left(c^{\prime}\right)^{2}\right]^{-1}, \\
\sin ^{2} \Theta=\frac{2}{3} \frac{2 f_{2}+c^{\prime}-f_{1} S^{-1}\left(1+c^{\prime}\right)}{2 f_{2}-c^{\prime} f_{1} S^{-1}} .
\end{gathered}
$$

In the $\operatorname{Sm} A$ phase the wave vector $k_{A}$ is expressed as

$$
k_{A}^{2}=k_{0}^{2}\left(1+f_{1} S^{-1}\right)\left(1+2 f_{2}+2 c^{\prime}\right)^{-1} .
$$

In the present model, the $\mathrm{Sm} A-\mathrm{Sm} C$ transition is governed by the temperature dependence of the nematic order parameter $S(T)$. One can readily see from Eq. (4) that

$$
\sin ^{2} \Theta \propto\left(S-S_{A C}\right),
$$

when $S$ exceeds the critical value

$$
S_{A C}=\frac{f_{1}\left(1+c^{\prime}\right)}{\left(2 f_{2}+c^{\prime}\right)}
$$

and the tilt angle vanishes when $S \leq S_{A C}$. Normally, the order parameter $S(T)$ is increasing with the temperature decreasing. For $S<S_{A C}$ the right-hand side of Eq. (4) is negative if $2 f_{2}>c^{\prime} f_{1} S^{-1}$, and hence there is no nonzero solution for $\Theta$. Thus the system is in the smectic- $A$ phase when $S(T)<S_{A C}$. The transition into the smectic- $C$ phase occurs when $S$ reaches the critical value $S_{A C}$. One notes that this mechanism of the $\operatorname{Sm} A-\operatorname{Sm} C$ transition is possible only if the order parameter $S$ is far from saturation. This condition is satisfied in many smectic- $C$ materials and, in particular, in de Vries smectics where $S$ is of the order of $0.6-0.7$ at the $\operatorname{Sm} A-\operatorname{Sm} C$ transition point [1].

In the $\operatorname{Sm} A$ phase [see Eq. (5)] the layer spacing $p_{A}$ $=2 \pi / k_{A}$ always increases for decreasing temperature; this is also true experimentally for practically all materials with low layer contraction [1]. In the $\mathrm{SmC}$ phase, the layer contraction is controlled by the parameter $c^{\prime}$. The spacing is constant for $c^{\prime}=0$, which can be regarded as ideal de Vries behavior. On the other hand, it follows from Eqs. (3), (4) that $k_{z}$

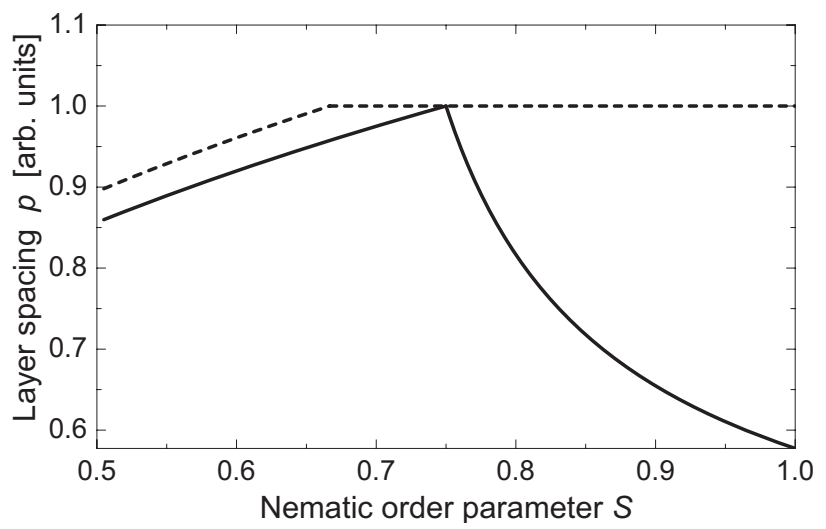

FIG. 1. Typical variation of smectic layer spacing versus nematic order parameter according to phenomenological formulas (3) and (5). Conventional contraction (solid line) is shown for $c^{\prime}=2$, $f_{1}=2$, and $f_{2}=3$. De Vries-like behavior (dashed line) corresponds to $c^{\prime}=0, f_{1}=2$, and $f_{2}=1.5$. 

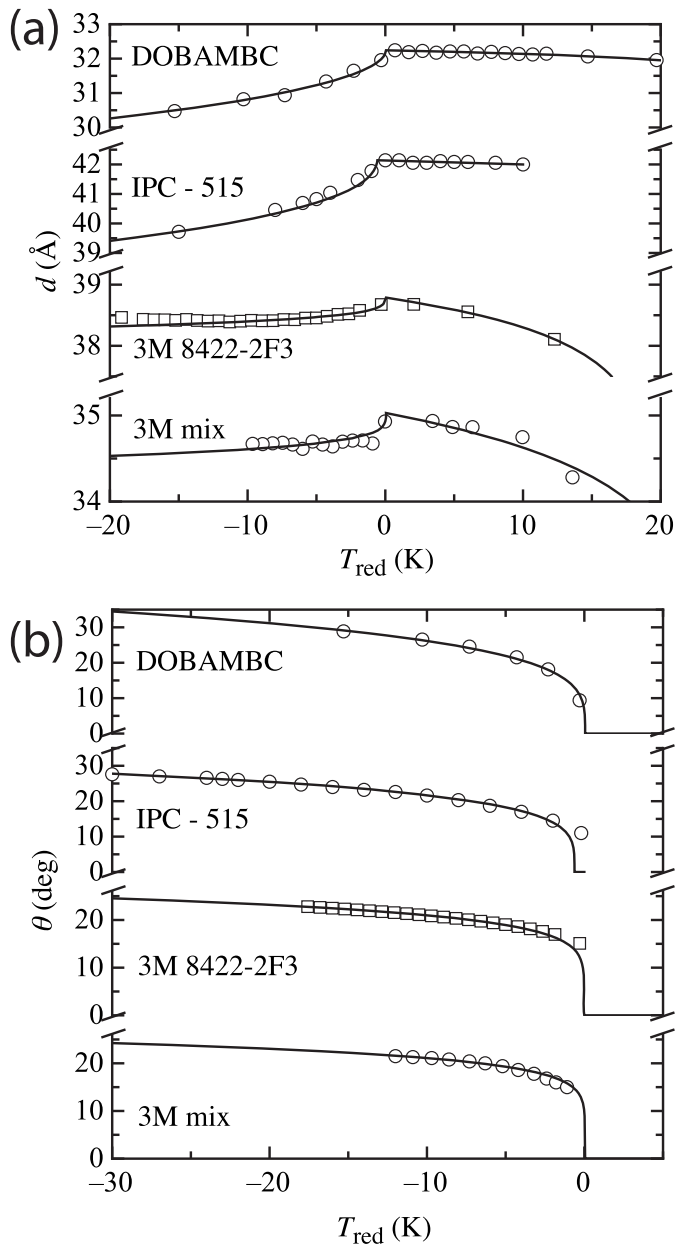

FIG. 2. Experimental data on temperature dependence of layer thickness (a) and tilt angle (b) for four different compounds fitted by the phenomenological relations (3)-(5). The parameters used are listed in Table I.

$=k_{C} \cos \Theta=$ const when $c^{\prime}=f_{2}-1$. In this case the layer spacing in the $\mathrm{SmC}$ phase decreases following the factor of $\cos \Theta$. This is ideal layer contraction in conventional smectics. Thus the simple phenomenological model describes both limiting cases which correspond to ideal de Vries and ideal conventional behavior, respectively. The dependence of the layer spacing on the order parameter $S$ in smectic- $A$ and smectic- $C$ phases for the two limiting cases is presented in Fig. 1.

Intermediate values of $c^{\prime}$ between 0 and $f_{2}-1$ correspond to a partial layer contraction in the smectic- $C$ phase which is observed experimentally for the majority of smectic materials. Simple model expressions (3)-(5) allow excellent fitting of experimental data for different materials of both de Vries (3M 8422-2F3 and 3M mixture) and conventional (IPC-515 and DOBAMBC) types as shown in Fig. 2 and Table I. One notes that the direct fitting of experimental data requires a model for the temperature variation of $S$. Here the parameter $S(T)$ has been approximated as $S_{A}(T) \propto\left(T_{A}-T\right)^{\beta_{A}}$ in the smectic- $A$ phase and $\left(S_{C}(T)-S_{A C}\right) \propto\left(T_{C}-T\right)^{\beta_{C}}$ where $S_{A C}$ is the value of $S$ at the $\operatorname{Sm} A-\operatorname{Sm} C$ transition point. This approximation takes into account an additional growth of $S$ in the smectic- $C$ phase induced by the tilt. This is justified by a
TABLE I. Parameters of phenomenological model used for fitting the data in Fig. 2.

\begin{tabular}{|c|c|c|c|c|}
\hline Parameter & $3 \mathrm{M} 8422-2 \mathrm{~F} 3$ & $3 \mathrm{M} \operatorname{mix}$ & IPC-515 & DOBAMBC \\
\hline$T_{A C}\left({ }^{\circ} \mathrm{C}\right)$ & 63.3 & 52.7 & 139.4 & 90.3 \\
\hline$c^{\prime}$ & 0.12 & 0.14 & 0.83 & 0.42 \\
\hline$f_{1}$ & 0.28 & 0.37 & 0.96 & 0.41 \\
\hline$f_{2}$ & 0.16 & 0.22 & 0.67 & 0.12 \\
\hline$k_{0}\left(\AA^{-1}\right)$ & 0.17 & 0.19 & 0.20 & 0.23 \\
\hline$S_{A C}$ & 0.70 & 0.72 & 0.81 & 0.89 \\
\hline$\beta_{A}$ & 0.13 & 0.12 & 0.16 & 0.05 \\
\hline$\beta_{C}$ & 0.32 & 0.29 & 0.48 & 0.53 \\
\hline
\end{tabular}

rapid growth of birefringence in the $\mathrm{SmC}$ phase of de Vries materials [1] and by the molecular theory developed in Sec. III B (see Fig. 5).

The phenomenological model (1) uses a free energy expansion in terms of the order parameter $S$ and components of the smectic wave vector, which in general are not small. Furthermore, the microscopic origin of the $\operatorname{Sm} A-\operatorname{Sm} C$ transition is not completely clear. To overcome these limitations we develop a molecular-statistical theory which is free of these approximations.

\section{MOLECULAR-STATISTICAL MODEL FOR THE SMECTIC-C PHASE}

\section{A. General results}

A molecular-statistical theory of the $\operatorname{Sm} A-\operatorname{Sm} C$ transition can be derived in the most general way using the densityfunctional approach to the theory of liquid crystals [39-41]. In this approach, the free energy of a liquid crystal $F$ is a functional of the one-particle distribution function $f$. For the uniaxial molecules, the distribution depends on molecular orientation $\omega$ and its position $\mathbf{r}$, i.e., $f=f(\omega, \mathbf{r})$. The general structure of the functional $F[f]$ is not known, but the functional derivatives are known and are related to the direct correlation functions in the medium. It is then possible to perform a functional Taylor expansion of the free energy of a liquid crystal phase around its value in the isotropic phase. The free energy of a smectic at temperature $T$ then can be written approximately as

$$
\begin{aligned}
F_{S}= & F_{I}+\rho k_{B} T \int f(\omega, \mathbf{r}) \ln [f(\omega, \mathbf{r}) \Lambda] d \omega d \mathbf{r} \\
& -\frac{1}{2} \rho^{2} k_{B} T \int C_{2}\left(\omega_{1}, \mathbf{r}_{1}, \omega_{2}, \mathbf{r}_{2}\right) \\
& \times \Delta f\left(\omega_{1}, \mathbf{r}_{1}\right) \Delta f\left(\omega_{2}, \mathbf{r}_{2}\right) d \omega_{1} d \omega_{2} d \mathbf{r}_{1} d \mathbf{r}_{2}+\cdots,
\end{aligned}
$$

where $F_{I}$ is the free energy of the isotropic phase, $\rho$ is the number density, $\Delta f(\omega, \mathbf{r})=f(\omega, \mathbf{r})-(4 \pi)^{-1}, \Lambda$ is a constant, and $C_{2}$ is the direct correlation function of the isotropic phase which is related to the full correlation function by the Ornstein-Zernike equation. The general expression for the free energy can be significantly simplified if one uses the 


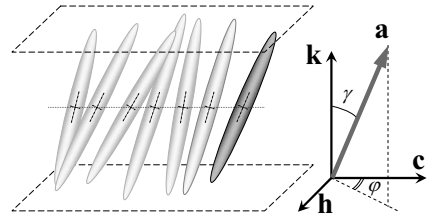

FIG. 3. Schematic of molecular orientation.

approximation of perfect translational order in the smectic- $C$ phase. In combination with the fact that there is no positional ordering within the layer, this means that the molecular distribution depends only on molecular orientation. For simplicity, we take into account that the tilt of the director is mainly determined by appropriate intermolecular interactions within the same smectic layer, while the interaction between different layers is of secondary importance here. Then the free energy per unit area of a single smectic layer can approximately be expressed as

$$
\begin{aligned}
\widetilde{F}_{S}= & \widetilde{F}_{I}+\rho_{2} k_{B} T \int f(\omega) \ln [f(\omega) \Lambda] d \omega \\
& -\frac{1}{2} \rho_{2} k_{B} T \int \breve{C}_{2}\left(\omega_{1}, \omega_{2}\right) f\left(\omega_{1}\right) f\left(\omega_{2}\right) d \omega_{1} d \omega_{2},
\end{aligned}
$$

where $\rho_{2}$ is the $2 \mathrm{D}$ number density (number of molecules per unit area of the layer) and

$$
\breve{C}_{2}\left(\omega_{1}, \omega_{2}\right)=\rho_{2} \int C_{2}\left(\omega_{1}, \mathbf{R}, \omega_{2}\right) d \mathbf{R} .
$$

The integration is performed over all intermolecular vectors $\mathbf{R}$ which are perpendicular to the smectic layer normal $\mathbf{k}$.

Minimization of the free energy (9) yields the Boltzmanntype orientational distribution function

$$
f(\omega)=\frac{1}{Z} \exp \left[-\frac{U_{1}(\omega)}{k_{B} T}\right],
$$

where $Z$ is the normalization constant and the effective oneparticle potential $U_{1}(\omega)$ is expressed as

$$
U_{1}(\omega)=-k_{B} T \int \breve{C}_{2}\left(\omega_{1}, \omega_{2}\right) f\left(\omega_{2}\right) d \omega_{2} .
$$

Consider smectic- $A$ and $C$ phases composed of rigid uniaxial molecules. In this case, the molecular orientation is given by the unit vector $\mathbf{a}$ in the direction of the molecular long axis (see Fig. 3). Then the direct correlation function $C_{2}$ depends on the unit vectors $\mathbf{a}_{1}$ and $\mathbf{a}_{2}$ which correspond to the molecules " 1 " and " 2 ," and on the intermolecular vector R. We consider nonchiral smectic phases, and thus the direct correlation function must be even in $\mathbf{R}, \mathbf{a}_{1}$, and $\mathbf{a}_{2}$ because the phases are also nonpolar. It is convenient to introduce the unit intermolecular vector $\hat{\mathbf{r}}=\mathbf{R} / R$. Then the direct pair correlation function $C_{2}\left(\mathbf{a}_{1}, \mathbf{R}, \mathbf{a}_{2}\right)$ may depend only on the intermolecular distance $R$ and on scalar products of the unit vectors $\mathbf{a}_{1}, \mathbf{a}_{2}, \hat{\mathbf{r}}$. As a result the correlation function can be expanded as

$$
\begin{aligned}
C_{2}\left(\mathbf{a}_{1}, \mathbf{R}, \mathbf{a}_{2}\right) \approx & v_{1}(R)\left[\left(\mathbf{a}_{1} \cdot \hat{\mathbf{r}}\right)^{2}+\left(\mathbf{a}_{2} \cdot \hat{\mathbf{r}}\right)^{2}\right]+v_{2}(R)\left(\mathbf{a}_{1} \cdot \mathbf{a}_{2}\right)^{2} \\
& +v_{3}(R)\left(\mathbf{a}_{1} \cdot \mathbf{a}_{2}\right)\left(\mathbf{a}_{1} \cdot \hat{\mathbf{r}}\right)\left(\mathbf{a}_{2} \cdot \hat{\mathbf{r}}\right) \\
& +v_{4}(R)\left(\mathbf{a}_{1} \cdot \hat{\mathbf{r}}\right)^{2}\left(\mathbf{a}_{2} \cdot \hat{\mathbf{r}}\right)^{2}
\end{aligned}
$$

where all possible terms quadratic in $\mathbf{a}_{1,2}$ have been taken into account.

Now the model direct correlation function (13) can be substituted into Eq. (10), integrated over $\mathbf{R}$ and then averaged over $\mathbf{a}_{2}$ to obtain the explicit expression for the effective one-particle potential (12). The averaging, however, is more explicit if the correlation function (13) is rewritten in terms of the molecular tensors $q_{i j}^{(1,2)}=\left(a_{1,2}\right)_{i}\left(a_{1,2}\right)_{j}-\delta_{i j} / 3$ :

$$
C_{2}\left(\mathbf{a}_{1}, \mathbf{R}, \mathbf{a}_{2}\right)=\left[\mathbf{q}^{(1)}+\mathbf{q}^{(2)}\right]: \mathbf{M}(\mathbf{R})+\mathbf{q}^{(1)}: \mathbf{N}(\mathbf{R}): \mathbf{q}^{(2)},
$$

where the tensors $\mathbf{M}$ and $\mathbf{N}$ read

$$
\begin{gathered}
M_{i j}(\mathbf{R})=\left[v_{1}(R)+\frac{1}{3}\left(v_{3}(R)+v_{4}(R)\right)\right] \hat{r}_{i} \hat{r}_{j}, \\
N_{i j k l}(\mathbf{R})=v_{2}(R) \delta_{i k} \delta_{j l}+v_{3}(R) \delta_{i k} \hat{r}_{j} \hat{r}_{l}+v_{4}(R) \hat{r}_{i} \hat{r}_{j} \hat{r}_{k} \hat{r}_{l} .
\end{gathered}
$$

One can readily evaluate $\breve{C}_{2}$ from Eq. (10). The integration over the unit intermolecular vector $\hat{\mathbf{r}}$ can be performed using the equations

$$
\begin{gathered}
\int d \hat{\mathbf{r}} \hat{r}_{i} \hat{r}_{j}=\pi T_{i j}, \\
\int d \hat{\mathbf{r}} \hat{r}_{i} \hat{r}_{j} \hat{r}_{k} \hat{r}_{l}=\frac{\pi}{4}\left(T_{i j} T_{k l}+T_{i k} T_{j l}+T_{i l} T_{j k}\right),
\end{gathered}
$$

where $T_{i j}=\delta_{i j}-k_{i} k_{j}$.

The averaging over $\mathbf{a}_{2}$ in Eq. (12) is reduced to the averaging of the molecular tensor $\mathbf{q}^{(2)}$, i.e., $\left\langle\mathbf{q}^{(2)}\right\rangle=\mathbf{Q}$, where $\mathbf{Q}$ is nematic tensor order parameter. Finally one obtains

$$
\begin{aligned}
U_{1}(\mathbf{a})= & -\frac{1}{2}\left(u_{1}+\frac{u_{3}}{3}+\frac{u_{4}}{3}\right) \mathbf{k} \cdot \mathbf{q} \cdot \mathbf{k}+\left(u_{2}+\frac{u_{3}}{2}+\frac{u_{4}}{4}\right) \mathbf{Q : q} \\
& -\frac{1}{2}\left(u_{3}+u_{4}\right) \mathbf{k} \cdot \mathbf{Q} \cdot \mathbf{q} \cdot \mathbf{k}+\frac{3}{8} u_{4}(\mathbf{k} \cdot \mathbf{Q} \cdot \mathbf{k})(\mathbf{k} \cdot \mathbf{q} \cdot \mathbf{k}),
\end{aligned}
$$

where we have introduced the constants $u_{\alpha}=$ $-\rho_{2} k_{B} T \int d \mathbf{R} v_{\alpha}(R)$.

The general expression for the tensor order parameter of the smectic- $C$ phase can be obtained directly by averaging the molecular tensor $\mathbf{q}$ taking into account the symmetry properties of the phase. In the general case, the traceless symmetric tensor $q_{i j}$ can be expressed as a sum of all irreducible orthogonal parts in the laboratory $(\mathbf{k}, \mathbf{c}, \mathbf{h})$ frame 


$$
\begin{aligned}
q_{i j}= & P_{2}(\cos \gamma)\left(k_{i} k_{j}-\delta_{i j} / 3\right)+\frac{1}{2} \sin ^{2} \gamma \cos 2 \varphi\left(c_{i} c_{j}-h_{i} h_{j}\right) \\
& +\frac{1}{2} \sin 2 \gamma \cos \varphi\left(c_{i} k_{j}+k_{i} c_{j}\right)+\frac{1}{2} \sin 2 \gamma \sin \varphi\left(h_{i} k_{j}+k_{i} h_{j}\right) \\
& +\frac{1}{2} \sin ^{2} \gamma \sin 2 \varphi\left(c_{i} h_{j}+h_{i} c_{j}\right),
\end{aligned}
$$

where the unit vector $\mathbf{c}$ is in the tilt plane and $\mathbf{h}$ is perpendicular to the tilt plane, the angles $\gamma$ and $\varphi$ specify the orientation of the unit vector $\mathbf{a}$ in the $(\mathbf{k}, \mathbf{c}, \mathbf{h})$ frame, i.e., $\mathbf{a}$ $=(\sin \gamma \cos \phi, \sin \gamma \sin \phi, \cos \gamma)$ (see also Fig. 3).

One notes that the last two terms in Eq. (20) are odd in $\varphi$ and their statistical averages vanish due to the mirror symmetry of the smectic- $C$ phase (invariance under $\varphi \rightarrow-\varphi$ ). Thus the orientational tensor order parameter $Q_{i j}$ can be expressed as

$$
Q_{i j}=S_{k}\left(k_{i} k_{j}-\delta_{i j} / 3\right)+\frac{1}{2} P_{k}\left(c_{i} c_{j}-h_{i} h_{j}\right)+\frac{1}{2} V\left(k_{i} c_{j}+c_{i} k_{j}\right),
$$

where $S_{k}, P_{k}$, and $V$ are the three independent orientational order parameters of the smectic- $C$ phase which can be explicitly expressed as the following statistical averages of the corresponding molecular quantities:

$$
\begin{gathered}
S_{k}=\left\langle P_{2}(\cos \gamma)\right\rangle, \quad P_{k}=\left\langle\sin ^{2} \gamma \cos 2 \varphi\right\rangle, \\
V=\langle\sin 2 \gamma \cos \varphi\rangle .
\end{gathered}
$$

Here the order parameter $S_{k}$ characterizes the tendency of long molecular axes to order along the smectic layer normal k. The order parameter $P_{k}$ is the nematic tensor biaxiality, i.e., it describes biaxial distribution of long molecular axes in the smectic plane. Finally, the tilt order parameter $V$ characterizes the nondiagonal terms in the $\mathbf{Q}$ tensor, i.e., the tilt of its main axis with respect to the layer normal $\mathbf{k}$.

Substituting Eqs. (20) and (21) into Eq. (19) one obtains the expression for the effective one-particle potential in terms of the order parameters $S_{k}, P_{k}, V$ :

$$
\begin{aligned}
U_{1}(\mathbf{a})= & w_{1} P_{2}(\cos \gamma)+w_{2} S_{k} P_{2}(\cos \gamma)+w_{3} P_{k} \sin ^{2} \gamma \cos 2 \phi \\
& +w_{4} V \sin 2 \gamma \cos \phi,
\end{aligned}
$$

where the parameters $w_{1-4}$ are linear combinations of the factors $u_{1-4}$ :

$$
\begin{gathered}
w_{1}=-u_{1} / 3-\left(u_{3}+u_{4}\right) / 9, \\
w_{2}=2 u_{2} / 3+\left(u_{3}+u_{4}\right) / 9, \\
w_{3}=u_{2} / 2+u_{3} / 4+u_{4} / 8, \\
w_{4}=u_{2} / 2+u_{3} / 8 .
\end{gathered}
$$

As discussed in the Appendix, the order parameters $S_{k}, P_{k}$, and $V$ can be easily related to the conventional order parameters of the smectic- $C$ phase appearing in the diagonal representation of the tensor $\mathbf{Q}$ :

$$
Q_{i j}=S\left(n_{i} n_{j}-\frac{1}{3} \delta_{i j}\right)+P\left(m_{i} m_{j}-h_{i} h_{j}\right) .
$$

The particular expressions for the tilt angle $\Theta$ and the order parameters $S$ and $P$ in terms of the order parameters $S_{k}, P_{k}$, and $V$ in the $\mathbf{k}$ frame are

$$
\begin{gathered}
\tan 2 \Theta=\frac{V}{S_{k}-0.5 P_{k}}, \\
S=\frac{1}{4} S_{k}+\frac{3}{8} P_{k}+\frac{3 V}{4 \sin 2 \Theta}, \\
P=\frac{1}{2} S_{k}+\frac{3}{4} P_{k}-\frac{V}{2 \sin 2 \Theta} .
\end{gathered}
$$

It should be noted that in the present theory the $\operatorname{Sm} A$ $\mathrm{Sm} C$ transition is described as an order-disorder one. The transition into the lower symmetry smectic- $C$ phase is signified by the emergence of the new order parameter $V$ which is described as a statistical average of the corresponding microscopic quantity. One can readily see from Eq. (30) that $V$ $\propto \Theta$ at small tilt angle $\Theta$. The biaxial order parameter $P_{k}$, which also vanishes in the smectic- $A$ phase, is the secondary order parameter of the smectic- $C$ phase which is induced by the tilt. $P_{k} \propto \Theta^{2}$ at small $\Theta \ll 1$. From the general point of view, the description of the $\operatorname{Sm} A-\operatorname{Sm} C$ phase transition in terms of a complete set of orientational order parameters $S_{k}, P_{k}, V$ explicitly defined as statistical averages, distinguishes the present approach from the previous models based on the tilt angle $\Theta$ as an order parameter which has a geometrical rather than a statistical definition. We note also that it is easier to measure the parameters $S_{k}, P_{k}$, and $V$ experimentally because they are defined with respect to the layer normal $\mathbf{k}$ which is usually well specified in the experiment (see, for example, Ref. [33]).

\section{B. $\operatorname{Sm} A-\operatorname{Sm} C$ phase transition}

The effective one-particle potential (24), derived in the previous subsection, depends on the three order parameters of the $\mathrm{SmC}$ phase, $S_{k}, P_{k}$, and $V$, and on the coupling constants $w_{1-4}$. These constants are the coefficients in the expansion of the direct correlation function. In a complex fluid, with both repulsion and attraction interaction the direct correlation function can be approximately expressed as

$$
C_{2}\left(\mathbf{a}_{1}, \mathbf{R}, \mathbf{a}_{2}\right)=C_{0}\left(\mathbf{a}_{1}, \mathbf{R}, \mathbf{a}_{2}\right)+\frac{1}{k_{B} T} U\left(\mathbf{a}_{1}, \mathbf{R}, \mathbf{a}_{2}\right),
$$

where

$$
U\left(\mathbf{a}_{1}, \mathbf{R}, \mathbf{a}_{2}\right)=\Theta\left(R-\xi_{12}\right) U_{\text {att }}\left(\mathbf{a}_{1}, \mathbf{R}, \mathbf{a}_{2}\right)
$$

is the pair attraction interaction potential $U_{\text {att }}$ modulated by the steric cutoff $\theta\left(R-\xi_{12}\right)$. Here $\xi_{12}$ is the minimum distance of approach between the centers of two rigid molecules with fixed mutual orientation. The step function $\theta\left(R-\xi_{12}\right)=0$ if the molecules penetrate each other, i.e., for $R<\xi_{12}$, and $\theta\left(R-\xi_{12}\right)=1$ otherwise. $C_{0}\left(\mathbf{a}_{1}, \mathbf{R}, \mathbf{a}_{2}\right)$ is the direct correlation 
function of the reference hard core system which in the limit of small molecular number density $\rho$ is reduced to the second virial approximation $C_{0}(1,2) \approx \theta(1,2)-1$.

In thermotropic liquid crystals, the phase transitions are governed by the change of temperature, which means that intermolecular attraction interaction is more important than pure steric repulsion [41]. Thus, we assume that the second term in Eq. (33) is predominant and thus the effective oneparticle potential (24) is temperature independent. Substituting Eqs. (11) and (24) into Eq. (9) one obtains the following explicit expression for the free energy:

$$
\tilde{F}_{S}=F_{0}-\frac{1}{2} \rho_{2}\left(w_{2} S_{k}^{2}+w_{3} P_{k}^{2}+w_{4} V^{2}\right)-\rho_{2} k_{B} T \ln Z,
$$

where

$$
Z=\int d \mathbf{a} \exp \left[-\frac{U_{1}(\mathbf{a})}{k_{B} T}\right]
$$

Now this free energy can be minimized numerically with respect to the order parameters $S_{k}, P_{k}$, and $V$ to locate the $\mathrm{Sm} A-\mathrm{Sm} C$ transition and to determine the temperature dependence of the order parameters.

One notes that the expansion of the direct correlation function (13) contains four different terms. The second term corresponds to the so-called Mayer-Saupe effective interaction potential $v_{2} P_{2}\left(\mathbf{a}_{1} \cdot \mathbf{a}_{2}\right)$ which is responsible for the nematic ordering. If one takes into account only this term, the coefficients $u_{1,3,4}=0$ and the parameter $w_{1}=0$, while $w_{3,4}$ $=3 / 4 w_{2}$. It is obvious that on its own the Mayer-Saupe interaction potential cannot induce the tilting transition because there is no coupling between molecular axes and layer normal. At the same time, taking into account other terms in Eq. (13) with small coefficients $v_{1,3,4}$ we obtain a distinctive tilting transition.

Two examples of the $\operatorname{Sm} A-\operatorname{Sm} C$ phase transition described by the current theory are presented in Fig. 4 where the calculated temperature variation of all order parameters in the $\operatorname{Sm} A$ and $\operatorname{Sm} C$ phases is shown. We have chosen $w_{2}$ $=-1$ to normalize the otherwise arbitrary temperature scale. Then the dimensionless constants $w_{3}$ and $w_{4}$ are chosen to be close to $-3 / 4$. One can readily see from Fig. 4 that the temperature variation of the tilt angle $\Theta$ is similar to that of the tilt order parameter $V$ although $\Theta$ is always smaller than $V$. Note that Figs. 4(a) and 4(b) correspond to the systems which differ only by the value of the parameter $w_{3}$. The temperatures of the $\operatorname{Sm} A-\operatorname{Sm} C$ transitions are exactly the same while the absolute values and temperature variation of the order parameters are different. Figure 4(b) describes a relatively weak transition with low tilt. In contrast, Fig. 4(a) corresponds to a strong tilting transition with steep variation of the tilt angle below the transition point and large values of the tilt. Both types of the transition have been observed in the experiment for different materials. In both cases, however, the biaxial order parameter $P$ is negligibly small. Thus the $\operatorname{Sm} A-\operatorname{Sm} C$ can be described as a simple rotation of the uniaxial distribution of long molecular axes. The nematic tensor order parameter remains uniaxial, and the nematic order parameter $S$ is practically not perturbed by the tilting.

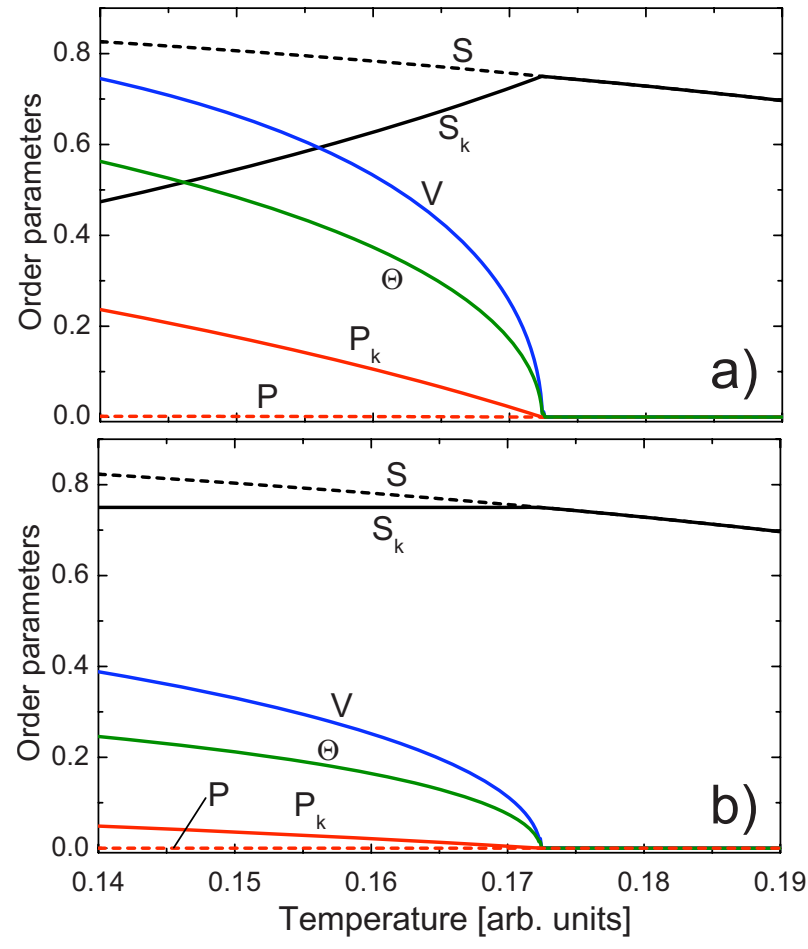

FIG. 4. (Color online) $\mathrm{Sm} A-\mathrm{Sm} C$ phase transition obtained for the parameters $w_{1}=-0.03, w_{2}=-1, w_{3}=-0.85$ (a) or $w_{3}=-0.78$ (b), $w_{4}=-0.78$.

Increasing the coefficients $u_{1}, u_{3}$, and $u_{4}$ and thus increasing the absolute values of $w_{1}$ and $\left(w_{3,4}+3 / 4\right)$ we obtain a stronger transition illustrated in Fig. 5. Here the transition occurs at lower value of the nematic order parameter $S$, and the nematic ordering is noticeably perturbed by the tilting transition. Such an increase of the nematic order just below the $\operatorname{Sm} A-\operatorname{SmC}$ transition point is indeed usually observed experimentally (see e.g., Figs. 5 and 6 in Ref. [42], as well as Ref. [31]).

Our general observation is that for all reasonable values of the parameters, the nematic tensor $\mathbf{Q}$ remains approximately uniaxial in the director frame, i.e., $P \ll 1$. This is in-line with the known experimental data [43] on the various order parameters of the smectic- $C$ phase revealed by the IR

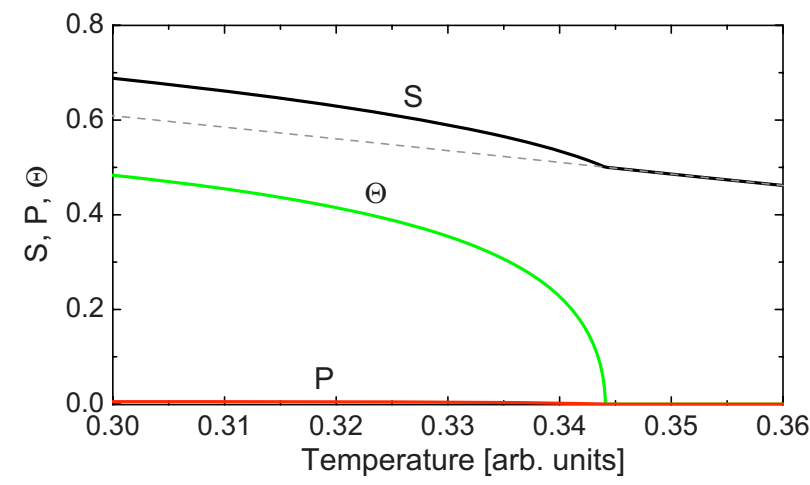

FIG. 5. (Color online) $\mathrm{Sm} A-\mathrm{Sm} C$ phase transition perturbing the nematic order. Thin dashed line shows the unperturbed $S$. The interaction constants are $w_{1}=-0.3, w_{2}=-1, w_{3}=-1.4, w_{4}=-1.2$. 
spectroscopy [44] and enables one to obtain simple analytical expressions for the $\mathrm{Sm} A-\mathrm{Sm} C$ transition temperature and the tilt angle.

Putting $P=0$ in Eqs. (30)-(32) one obtains

$$
S_{k}=S P_{2}(\cos \Theta), \quad P_{k}=S \sin ^{2} \Theta, \quad V=S \sin 2 \Theta .
$$

Next, these simple expressions can be substituted into the free energy (35), and the free energy can then be minimized with respect to $\Theta$. The tilt angle $\Theta$ plays the role of the parameter in the free energy, and it can be shown that minimization of the free energy with respect to such a parameter is equivalent to minimization the averaged potential $\left\langle U_{1}(S, \Theta)\right\rangle$, which yields

$$
\begin{aligned}
& \sin 2 \Theta\left[\sin ^{2} \Theta S\left(9 w_{2}+4 w_{3}-16 w_{4}\right)-6 w_{1}-6 S w_{2}+4 S w_{4}\right] \\
& \quad=0 .
\end{aligned}
$$

One can readily see that in addition to the trivial solution $\Theta=0$, this equation acquires the nonzero solution for $\Theta$ when $S$ exceeds

$$
S_{A C}=\frac{3 w_{1}}{4 w_{4}-3 w_{2}} .
$$

Thus in the present model the $\operatorname{Sm} A-\operatorname{Sm} C$ transition occurs when the nematic order parameter reaches the critical value $S_{A C}$. The transition temperature can be obtained from the equation $S(T)=S_{A C}$, where $S(T)$ is the temperature variation of $S$ in the smectic- $A$ phase, and the value of $S_{A C}$ is specified by the parameters $w_{1}, w_{2}$, and $w_{4}$. The transition temperature calculated in this way exactly coincides with that found by numerical minimization of the free energy. Equation (39) also explains our observation that the transition temperature is not affected by a change in $w_{3}$ (as seen from Fig. 4), since the critical value $S_{A C}$ is simply independent of the constant $w_{3}$.

Below the transition point, the tilt angle is given by

$$
\sin ^{2} \Theta=\frac{S-S_{A C}}{S S_{A C}} \frac{6\left|w_{1}\right|}{9 w_{2}+4 w_{3}-16 w_{4}} .
$$

Thus the temperature variation of the tilt in this model is determined by that of the nematic order parameter $S$. It should be noted that this equation does not specify the temperature variation of the tilt angle in a simple way because the nematic order parameter $S$ is also affected by the tilt. Nevertheless, Eq. (40) can be used to understand the role of different parameters and to describe the temperature variation of the tilt directly in the case of relatively large $S$ when the influence of the tilt is small. One notes, for instance, that the tilt angle increases with the increasing absolute value $\left|w_{3}\right|$, which is again confirmed by our numerical calculations.

It is interesting to describe the variation of the tilt analytically for the case of weak tilting transition which does not perturb the order parameter $S$. The function $S(T)$ can be expanded in the vicinity of the transition temperature giving $\left(S-S_{A C}\right) \propto\left(T_{A C}-T\right)$, and Eq. (40) yields the classical behavior of the tilt angle $\Theta \propto \sqrt{T_{A C}-T}$. At the same time, this is the only reasonable limit, in which this classical temperature dependence predicted by simple phenomenological models can

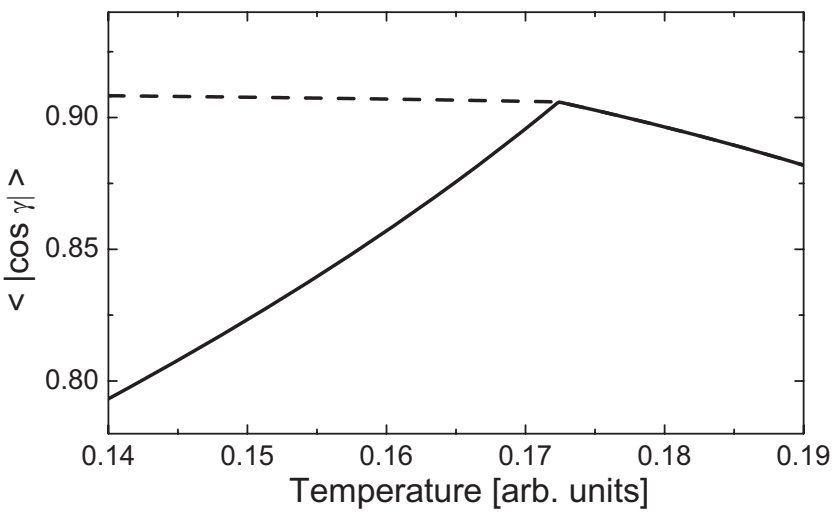

FIG. 6. Temperature dependence of the average projection of long molecular axis on the layer normal. The interaction constants are taken as in Fig. 4(a) (solid line) and Fig. 4(b) (dashed line).

be obtained. Although the numerically calculated dependence $\Theta(T)$ can be well fitted by the power law $\left(T_{A C}-T\right)^{\nu}$ with the index $\nu$ ranging from $1 / 2$ to $1 / 4$, the particular values of $\nu$ do not have any physical meaning because they are not universal and are determined by a coupling between the two order parameters $\Theta$ and $S$.

\section{Weak layer contraction}

Let us now explore how the tilting transition affects the thickness of smectic layers. Although our model does not enable one to obtain the layer spacing directly, we can extract important quantitative information from the calculated orientational distribution function. Indeed, the average projection of the molecular unit vector onto the layer normal $\langle|\cos \gamma|\rangle$ can be used as a suitable surrogate reflecting the variations of the layer thickness [45]. This intuitive assumption is, in fact, strongly supported by recent experimental results. It has been shown [33] that for several different smectic- $C$ materials there exists a good correlation between the temperature variation of the smectic periodicity $p$ and the orientational order parameter $S_{k}=1-3 / 2\left\langle\sin ^{2} \gamma\right\rangle$. On the other hand, the temperature variation of $S_{k}$ should be qualitatively similar to that of the average projection of the molecular long axes $\langle|\cos \gamma|\rangle$. Indeed, for a relatively high orientational order parameter $S$

$$
\langle|\cos \gamma|\rangle \approx 1-0.5\left\langle\sin ^{2} \gamma\right\rangle \text {, i.e., }\langle|\cos \gamma|\rangle \approx\left(2+S_{k}\right) / 3,
$$

and therefore the changes in $S_{k}$ are proportional to those in the projection $\langle|\cos \gamma|\rangle$.

Temperature variation of the layer spacing evaluated for the same two systems as in Fig. 4 is presented in Fig. 6. One can readily see that while the increase in the tilt angle promotes the decrease of the layer thickness, the enhancement of the nematic order below the transition point leads to an increase in the spacing. As a result, the contraction can be weak or even absent if the tilting is not strong enough, and the two factors approximately compensate each other. This result suggests a simple explanation of weak layer contraction observed in de Vries-like materials with low tilt [23]. 


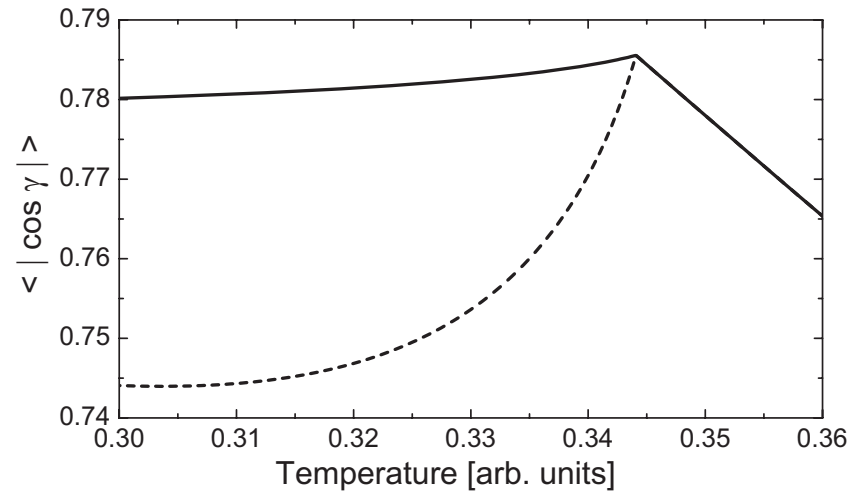

FIG. 7. The effect of the perturbation of nematic order on the layer spacing. The solid line shows the real behavior with constants as in Fig. 5, while the dashed line is calculated according to the classical formula (42).

At the same time, we obtain anomalously weak layer contraction in a number of cases with a strong tilt. A typical example is presented in Fig. 7, which corresponds to the same system as Fig. 5. In Fig. 7 the temperature variation of the layer spacing calculated numerically is compared with that expected from the classical relation

$$
p_{C}(T)=p_{A}(T) \cos \Theta .
$$

Here the layer spacing $p_{A}(T)$ can be found by extrapolation from the $\operatorname{Sm} A$ phase. In our numerical calculations the temperature dependence of $p_{A}(T)$ is directly obtained by suppressing the tilting transition.

An apparent reason for the anomalously low layer contraction seen in Fig. 6 is the rapid increase of nematic order below the transition temperature, which compensates the decrease in thickness caused by the tilting. Remarkably, the stronger is the tilting the more differs the thickness from the classical dependence (42).

This is how the weak layer contraction can be understood both in the case of low and high tilt. On the other hand, such behavior is not obtained for all systems, and some special conditions must be satisfied. The relations (37) make it possible to derive a simple analytical condition on the effective interaction potential, which is sufficient to generate an approximately constant layer spacing in the $\mathrm{SmC}$ phase. Firstly we take into consideration that the temperature variation of the layer spacing is qualitatively similar to that of the order parameter $S_{k}$, as discussed above. One notes that the ideal classical temperature variation of $S_{k}$ decreasing by the factor $\cos \Theta$ can be obtained only in the case of a constant order parameter $S$. This is only possible if $S$ is large and close to saturation, i.e., $S \approx 1$. In contrast, in the case of relatively low $S$, which is typical for de Vries materials, the decrease of $S_{k}$ (and thus the decrease of the layer spacing) in the $\mathrm{SmC}$ phase, determined by the factor of $P_{2}(\cos \Theta)$, is at least partially compensated by the growth of $S$. This also explains why the ideal classical layer contraction is rarely observed. The explicit condition which corresponds to a constant layer spacing can be obtained by substituting (40) into the expression for $S_{k}$ from (37), which yields

$$
S_{k}=S_{A C}+\left(S-S_{A C}\right) \frac{w_{3}-w_{4}}{9 w_{2} / 4+w_{3}-4 w_{4}} .
$$

One can readily see that $S_{k}=S_{A C}=$ const if $w_{3}=w_{4}$. This condition is supported by our numerical calculations as can be seen from Fig. 4(b) and the dashed line in Fig. 6.

Note that in contrast to the original qualitative model of de Vries, our explanation of the anomalously weak layer contraction does not involve any additional assumptions about the specific form of the orientational distribution function in the $\operatorname{Sm} A$ phase, which would correspond to a cone model with a constant molecular tilt made by de Vries. In the present theory, both conventional and de Vries smectics $C$ are described by the same molecular model, and the weak layer contraction simply corresponds to a particular relationship between the coupling constants in the effective interaction potential combined with the condition of relatively low values of the nematic order parameter $S$ which leads to a constant projection of the molecular orientational distribution on the smectic layer normal.

\section{Tricritical behavior at the transition}

Many conventional smectic materials undergo the $\operatorname{Sm} A$ $\mathrm{Sm} C$ transition according to the classical second order scenario with no temperature hysteresis and small discontinuity of the heat capacity $c_{p}(T)$ at the transition point. On the other hand, in a number of de Vries-type materials the transition is often concluded to be weakly first order on the basis of sharp peaks on the $c_{p}(T)$ curve. At the same time, the measured temperature interval of the phase coexistence is extremely small, of the order of $10 \mathrm{mK}[34,42]$. In this subsection we show that these experimental data can also be interpreted in the framework of the present model by considering the second order $\operatorname{Sm} A-\operatorname{Sm} C$ transitions which are close to the tricritical point.

Let us first consider the temperature variation of the heat capacity predicted by the present model. The orientational entropy of the smectic liquid crystal can be evaluated as

$$
\mathcal{S}=-\langle\ln f(\mathbf{a})\rangle
$$

using the distribution function (11) and the effective one particle potential (24) with the order parameters obtained from the free energy minimization. Now the heat capacity can be expressed as

$$
c_{p}(T)=k_{B} T \frac{\partial \mathcal{S}}{\partial T} .
$$

Temperature variation of the heat capacity which corresponds to the classical transitions presented in Fig. 4 is shown in Fig. 8. The behavior of $c_{p}$ is typical to a second order phase transition. The amplitude of the discontinuity of the heat capacity is directly related to the tilting amplitude. The transition with lower tilt and lower layer contraction makes a smaller effect on the variation of the heat capacity.

A different result is obtained when the transition is accompanied by a noticeable variation of the nematic order parameter. The coupling between $\Theta$ and $S$ results in a quali- 


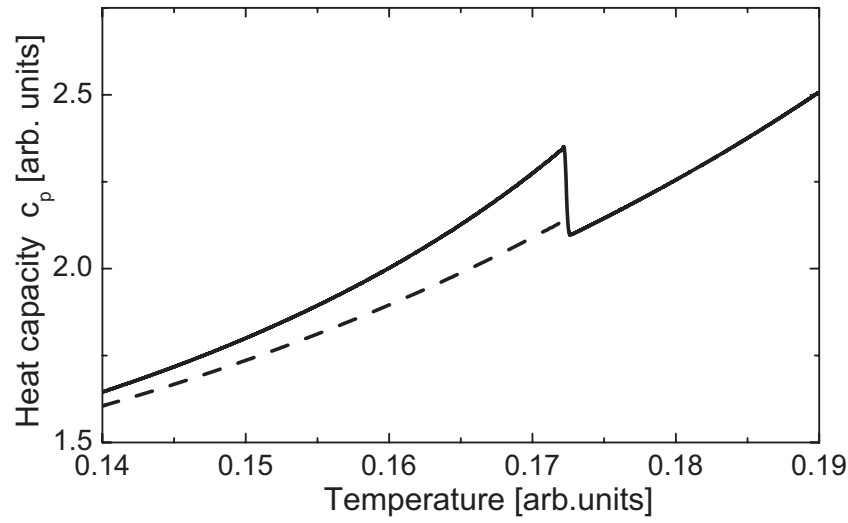

FIG. 8. Discontinuity of the heat capacity due to a conventional second order $A-C$ transition. The interaction constants are the same as in Figs. 4(a) (solid line) 4(b) (dashed line).

tative change of the $c_{p}(T)$ dependence. Here the discontinuity is accompanied by a pronounced peak of the heat capacity as illustrated in Fig. 9. The stronger is the nematic ordering affected by the tilting transition, the higher is the $c_{p}(T)$ peak at the transition point. One notes that the shape of the curve resembles the experimentally observed first-order-like anomaly of the heat capacity [34], although in the present model the transition always remains continuous. This behavior may be observed when the transition is close to the tricritical point.

Thus one concludes that the coupling of tilting and nematic order parameters can be responsible for both weak layer contraction and heat capacity anomaly, which are frequently observed in the same materials. These phenomena may also occur independently if the weak layer contraction is mainly due to a low tilt, or if the perturbation of the nematic order is too weak to compensate the layer shrinkage.

\section{INTERMOLECULAR INTERACTIONS}

The results obtained in the previous section do not depend on any particular molecular model because the theory is based on a general expansion of the direct correlation func-

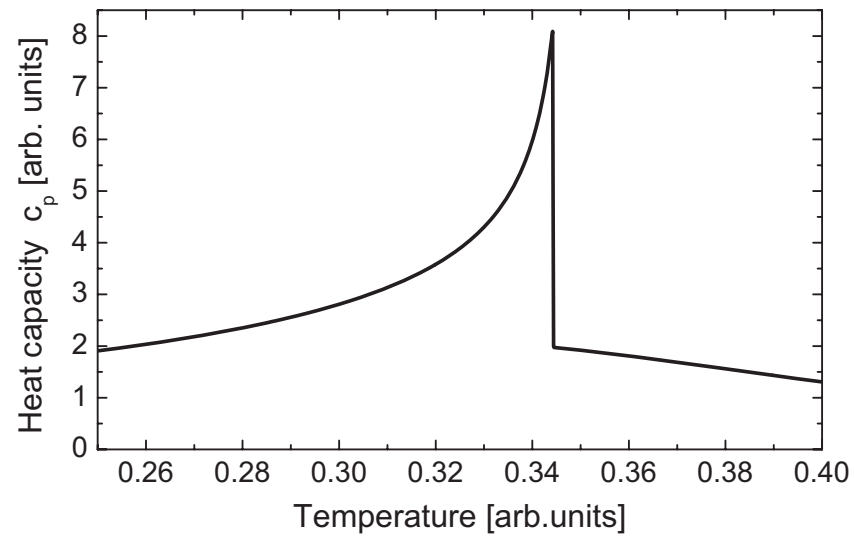

FIG. 9. Anomaly of heat capacity due to a perturbation of the nematic order by the tilting transition. The interaction constants are the same as in Figs. 5 and 7. tion (13). In this section we discuss how the coefficients in such an expansion can be evaluated for particular models of the intermolecular interaction, which determine $C_{2}$ according to Eq. (33). First, let us demonstrate how Eq. (24) can be derived in an alternative way based on the symmetry properties of the pair correlation function.

Let us consider the effective pair potential from Eq. (33) integrated over the intermolecular vector $\mathbf{R}$ within the smectic plane as implied by Eq. (10):

$$
\widetilde{U}\left(\mathbf{a}_{1}, \mathbf{a}_{2}\right)=\rho_{2} \int d \mathbf{R} U\left(\mathbf{a}_{1}, \mathbf{R}, \mathbf{a}_{2}\right) .
$$

The potential $\tilde{U}$ depends on the polar angles $\gamma_{1}, \gamma_{2}$ and the absolute value of the difference of the azimuthal angles, $\phi$ $=\varphi_{2}-\varphi_{1}$. Thus it can be expressed as the following series of spherical harmonics:

$$
\begin{aligned}
\tilde{U}\left(\gamma_{1}, \gamma_{2}, \phi\right)= & \sum_{n=0}^{\infty} \sum_{l=0}^{\infty} \sum_{m=0}^{\min (n, l)} U_{l n}^{(m)} P_{n}^{(m)}\left(\cos \gamma_{1}\right) \\
& \times P_{l}^{(m)}\left(\cos \gamma_{2}\right) \cos (m \phi),
\end{aligned}
$$

where $P_{\alpha}^{(\beta)}$ are the associated Legendre polynomials. For a given particular $\tilde{U}$, the expansion coefficients can be evaluated as the following integrals:

$$
\begin{aligned}
U_{l n}^{(m)}= & \frac{(2 n+1)(2 l+1)(l-m) !(n-m) !}{4 \pi(l+m) !(n+m) !} \\
& \times \int_{0}^{\pi} d \gamma_{1} \sin \gamma_{1} P_{n}^{(m)}\left(\cos \gamma_{1}\right) \\
& \times \int_{0}^{\pi} d \gamma_{2} \sin \gamma_{2} P_{l}^{(m)}\left(\cos \gamma_{2}\right) \\
& \times \int_{0}^{2 \pi} d \phi \cos (m \phi) \tilde{U}\left(\gamma_{1}, \gamma_{2}, \phi\right) .
\end{aligned}
$$

In a nonpolar liquid crystal phase the molecular heads and tails are statistically equivalent and thus the effective interaction potential has to be invariant under the transformation $\theta_{i} \rightarrow \theta_{i}+\pi$, which excludes the terms with odd $n$ and $l$ from Eq. (47). The permutational symmetry $1 \leftrightarrow 2$ yields that $U_{l n}^{(m)}=U_{n l}^{(m)}$.

It is reasonable to assume that the thermodynamics of the orientationally ordered phases is not governed by higher harmonics of the expansion corresponding to higher rank orientational order parameters. Our aim is to show that it is sufficient to take into account only the terms with lower $l, n, m$ to obtain realistic scenarios of the $\operatorname{Sm} A-\operatorname{Sm} C$ phase transition described in the previous section. If we truncate the series and keep the harmonics with $l, n \leq 2$, the remaining terms can be expressed as

$$
\begin{aligned}
\tilde{U}\left(\gamma_{1}, \gamma_{2}, \phi\right)= & w_{1}\left[P_{2}\left(\cos \gamma_{1}\right)+P_{2}\left(\cos \gamma_{2}\right)\right] \\
& +w_{2} P_{2}\left(\cos \gamma_{1}\right) P_{2}\left(\cos \gamma_{2}\right) \\
& +w_{3} \sin ^{2} \gamma_{1} \sin ^{2} \gamma_{2} \cos 2 \phi \\
& +w_{4} \sin 2 \gamma_{1} \sin 2 \gamma_{2} \cos \phi
\end{aligned}
$$


a)

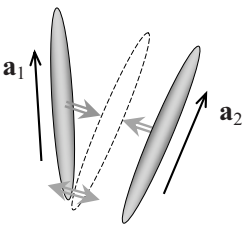

b)
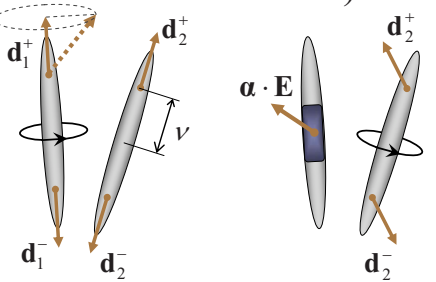

FIG. 10. (Color online) Schematics of model pair molecular interactions: hardcore repulsion attraction (a), electrostatic dipoledipole coupling (b), and dipolar induction interaction (c).

where the constants

$$
w_{1}=U_{20}^{(0)}, \quad w_{2}=U_{22}^{(0)}, \quad w_{3}=9 U_{22}^{(2)}, \quad w_{4}=-9 / 4 U_{22}^{(1)}
$$

can readily be calculated using Eq. (48).

Now the effective one-particle potential can be evaluated by averaging of Eq. (49) over the orientation of the molecule "2." Choosing the $c$ axis along the direction of the tilt in the $\operatorname{Sm} C$ phase one obtains that $\left\langle\sin \left(\varphi_{2}\right)\right\rangle=\left\langle\sin \left(2 \varphi_{2}\right)\right\rangle=0$, and the resulting expression for the effective potential takes exactly the form of Eq. (24).

Therefore, we have developed a procedure which enables one to trace the relation between the intermolecular interaction potential and the thermodynamic properties of smectic- $A$ and $-C$ phases. Below we illustrate this using simple interaction potentials.

\section{A. Gay-Berne potential}

In liquid crystals, the main part of pair interaction potential is determined by the short-range attraction and repulsion of hard elongated molecules [see Fig. 10(a)]. Among existing models, the Gay-Berne (GB) potential $[46,47]$ is popular for its relative simplicity and capability of reproducing orientational order of anisotropic liquids $[47,48]$.

The GB potential expresses the pair intermolecular interaction energy in the Lenard-Jones form

$$
\begin{aligned}
U_{\mathrm{GB}}\left(\mathbf{a}_{1}, \mathbf{R}, \mathbf{a}_{2}\right)= & 4 \varepsilon\left(\mathbf{a}_{1}, \hat{\mathbf{r}}, \mathbf{a}_{2}\right)\left\{\left[R / r_{0}-\sigma\left(\mathbf{a}_{1}, \hat{\mathbf{r}}, \mathbf{a}_{2}\right)+1\right]^{-12}\right. \\
& \left.-\left[R / r_{0}-\sigma\left(\mathbf{a}_{1}, \hat{\mathbf{r}}, \mathbf{a}_{2}\right)+1\right]^{-6}\right\}
\end{aligned}
$$

with the orientationally dependent range

$$
\begin{aligned}
\sigma\left(\mathbf{a}_{1}, \hat{\mathbf{r}}, \mathbf{a}_{2}\right)= & {\left[1-\frac{\chi}{2}\left(\frac{\left(\hat{\mathbf{r}} \cdot \mathbf{a}_{1}+\hat{\mathbf{r}} \cdot \mathbf{a}_{2}\right)^{2}}{1+\chi \mathbf{a}_{1} \cdot \mathbf{a}_{2}}\right.\right.} \\
& \left.\left.+\frac{\left(\hat{\mathbf{r}} \cdot \mathbf{a}_{1}-\hat{\mathbf{r}} \cdot \mathbf{a}_{2}\right)^{2}}{1-\chi \mathbf{a}_{1} \cdot \mathbf{a}_{2}}\right)\right]^{-1 / 2}
\end{aligned}
$$

and strength

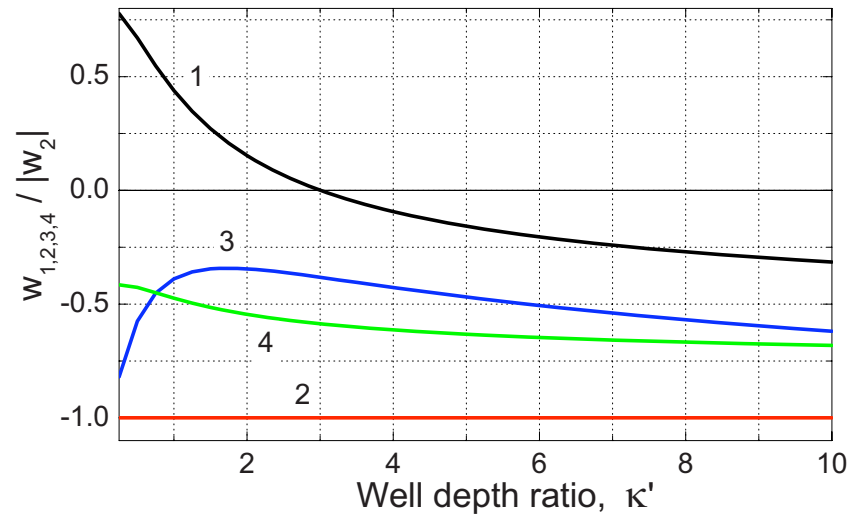

FIG. 11. (Color online) The ratios $w_{i} /\left|w_{2}\right|$ as a function of well depth anisotropy calculated for the Gay-Berne potential with the molecular elongation $\kappa=4$. The indices $i$ are indicated.

$$
\begin{aligned}
\varepsilon\left(\mathbf{a}_{1}, \hat{\mathbf{r}}, \mathbf{a}_{2}\right)= & \varepsilon_{0}\left[1-\chi^{2}\left(\mathbf{a}_{1} \cdot \mathbf{a}_{2}\right)^{2}\right]^{-1 / 2} \\
& \times\left[1-\frac{\chi^{\prime}}{2}\left(\frac{\left(\hat{\mathbf{r}} \cdot \mathbf{a}_{1}+\hat{\mathbf{r}} \cdot \mathbf{a}_{2}\right)^{2}}{1+\chi^{\prime} \mathbf{a}_{1} \cdot \mathbf{a}_{2}}\right.\right. \\
& \left.\left.+\frac{\left(\hat{\mathbf{r}} \cdot \mathbf{a}_{1}-\hat{\mathbf{r}} \cdot \mathbf{a}_{2}\right)^{2}}{1-\chi^{\prime} \mathbf{a}_{1} \cdot \mathbf{a}_{2}}\right)\right]^{2} .
\end{aligned}
$$

Here $r_{0}$ is the breadth of the molecule, and the constants $\chi$ $=\left(\kappa^{2}-1\right) /\left(\kappa^{2}+1\right)$ and $\chi^{\prime}=\left(\kappa^{\prime 1 / 2}-1\right) /\left(\kappa^{\prime 1 / 2}+1\right)$ are determined by the relative elongation of the molecule $\kappa$ as well as by the ratio $\kappa^{\prime}$ of well depths for side-to-side and end-to-end molecular orientations.

In accordance with Eq. (34) we multiply the GB potential by the steric cutoff $\theta\left[R-r_{0} \sigma\left(\mathbf{a}_{1}, \hat{\mathbf{r}}, \mathbf{a}_{2}\right)\right]$, substitute this into Eqs. (46)-(50), integrate numerically, and finally obtain the parameters of the corresponding one particle potential.

A typical dependence of the normalized values of the constants $w_{1-4}$ on the well depth $\kappa$ is presented in Fig. 11. As expected, the Gay-Berne interaction does stabilize the nematic order in the $\operatorname{Sm} A$ phase because all constants are negative, but it does not promote the tilting phase transition. The $\mathrm{Sm} A-\mathrm{SmC}$ transition can only occur if the constant $w_{4}$ is smaller than $3 w_{2} / 4$ [as follows from the condition $S_{A C}>0$ in Eq. (39)], which does not happen for any set of the model parameters.

\section{B. Electrostatic dipole-dipole interaction}

The electrostatic interaction between anisotropic molecules can qualitatively be described in terms of static and induced multipole moments of various molecular fragments. For electrically neutral but polar molecules, the dipolar interactions are usually predominant and are expected to contribute substantially to the effective interaction potential. Let us first consider the electrostatic interaction between two permanent dipoles $\mathbf{d}_{1}$ and $\mathbf{d}_{2}$ of the molecules 1 and 2, where the dipole $\mathbf{d}=d_{\|} \mathbf{a}+d_{\perp} \mathbf{b}$ has an arbitrary orientation in the molecular frame and is located on the axis of the uniaxial molecule. After averaging over all orientations of the short molecular axis $\mathbf{b}$ and the states with $+\mathbf{a}$ and $-\mathbf{a}$ (head-tail invariance), the arbitrary off-center dipole is effectively 


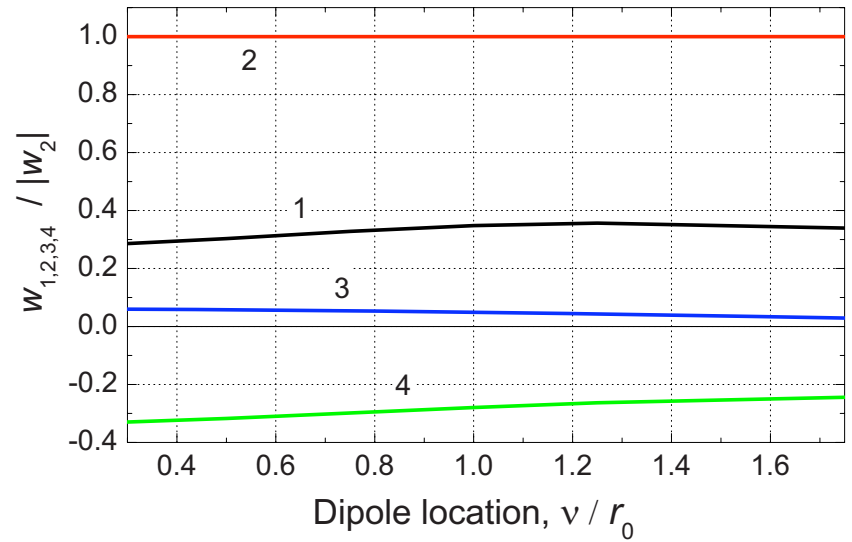

FIG. 12. (Color online) Normalized coefficients $w_{i} /\left|w_{2}\right|, i$ $=1,2,3,4$ arising from the dipole-dipole electrostatic potential as a function of the dipole location $\nu$ calculated with the hard rod cutoff $\kappa=4$.

transformed into a symmetric pair of antiparallel longitudinal dipoles [see Fig. 10(b)], and the resulting interaction is expressed as a sum of four dipole-dipole terms

$$
\begin{aligned}
U_{\mathrm{dip}}\left(\mathbf{a}_{1}, \mathbf{R}, \mathbf{a}_{2}\right)= & U_{d d}\left(\mathbf{d}_{1}^{+}, \mathbf{d}_{2}^{+}, \mathbf{r}_{1}^{+}-\mathbf{r}_{2}^{+}\right)+U_{d d}\left(\mathbf{d}_{1}^{+}, \mathbf{d}_{2}^{-}, \mathbf{r}_{1}^{+}-\mathbf{r}_{2}^{-}\right) \\
& +U_{d d}\left(\mathbf{d}_{1}^{-}, \mathbf{d}_{2}^{+}, \mathbf{r}_{1}^{-}-\mathbf{r}_{2}^{+}\right)+U_{d d}\left(\mathbf{d}_{1}^{-}, \mathbf{d}_{2}^{-}, \mathbf{r}_{1}^{-}-\mathbf{r}_{2}^{-}\right),
\end{aligned}
$$

where the single dipole-dipole interaction has the standard form

$$
U_{d d}\left(\mathbf{d}, \mathbf{d}^{\prime}, \mathbf{r}\right)=-r^{-5}\left[3(\mathbf{r} \cdot \mathbf{d})\left(\mathbf{r} \cdot \mathbf{d}^{\prime}\right)-r^{2}\left(\mathbf{d} \cdot \mathbf{d}^{\prime}\right)\right],
$$

the interacting dipoles are parallel to the long axes, $\mathbf{d}_{i}^{ \pm}$ $= \pm d_{\|} / 2 \mathbf{a}_{i}$ and located at $\mathbf{r}_{i}^{ \pm}=\mathbf{r}_{i} \pm \nu \mathbf{a}_{i}$.

The coefficients of the effective one-particle potential arising from the dipole-dipole interaction are again obtained numerically using Eqs. (46)-(50) and the same GB cutoff in Eq. (34) as above. We have found that the absolute values of the coefficients grow almost linearly with the distance $\nu$. At the same time, the ratios of different coefficients $w_{i}$ remain practically constant as illustrated in Fig. 12. The obvious feature of the dipole-dipole interaction is the negative value of the constant $w_{4}$, while all other constants are positive. Thus, the combination of the dipole-dipole interaction with the reference GB potential may be responsible for the $\operatorname{Sm} A$ $\mathrm{SmC}$ phase transition. The temperature variation of the order parameters around the transition caused by the electrostatic dipole-dipole interactions are presented in Fig. 13 for a particular value of the molecular dipole.

One notes that the $\mathrm{SmC}$ phase stabilized by electrostatic interaction between pairs of antiparallel molecular dipoles has been found in computer simulations [15]. According to Ref. [15] the longitudinal molecular dipoles appear to be much more effective in stabilizing the $\mathrm{SmC}$ phase than the transverse ones, which supports the present model. One notes that the far field of the pair of molecular dipoles coincides with that of an effective quadrupole. Therefore, our results also correspond to the case when the tilting transition

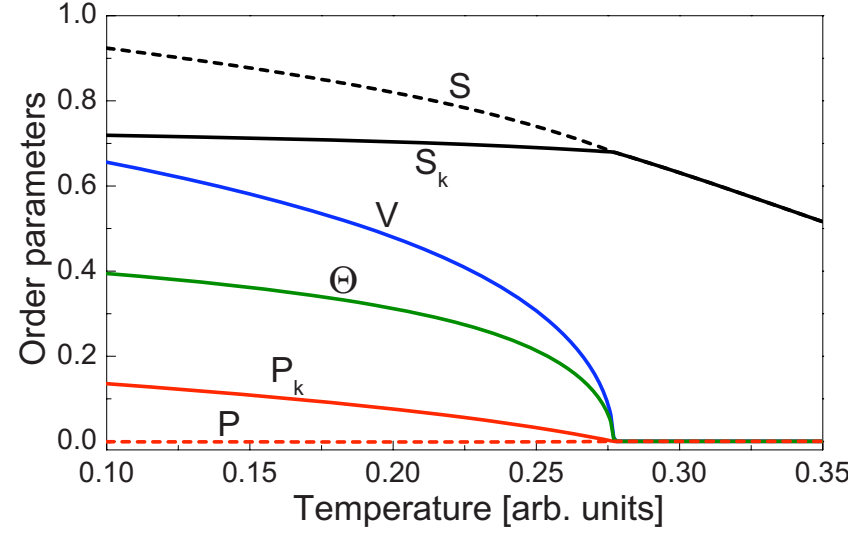

FIG. 13. (Color online) Example of the $\operatorname{Sm} A-\operatorname{Sm} C$ phase transition occurring when the dipole-dipole interaction is added to the Gay-Berne potential. The parameters were chosen to provide the dimensionless ratio $\frac{d^{2} v}{r_{0}^{4} \varepsilon_{0}}=0.11$.

is caused by the quadrupole-quadrupole interactions. This effect has also been observed in computer simulation [14].

\section{Dipolar induction interaction}

Another strong dipolar interaction is the induction interaction between a permanent molecular dipole and the polarizability of neighboring molecule. We assume for simplicity that the polarizable core is located in the middle of the molecule, and the main axis of the polarizability tensor $\alpha$ coincides with the long molecular axis a:

$$
\alpha_{\mu \nu}=\alpha_{\|} a_{\mu} a_{\nu}+\alpha_{\perp}\left(\delta_{\mu \nu}-a_{\mu} a_{\nu}\right) .
$$

The permanent molecular dipole has an arbitrary orientation [see Fig. 10(c)].

Similarly to the case of the electrostatic dipole-dipole interaction, averaging over all orientations of the short molecular axis $\mathbf{b}$ and the opposite directions of the long axis $\mathbf{a}$ results in four induced dipole-dipole contributions to the total pair interaction potential. All the four contributions have the same mathematical form. For example, the contribution related to the $\mathbf{d}_{2}^{+}$dipole is expressed as

$$
\begin{aligned}
U_{2}^{+}\left(\mathbf{a}_{1}, \mathbf{R}, \mathbf{a}_{2}\right)= & \eta_{1} \frac{\left(\mathbf{a}_{1} \cdot \mathbf{R}_{2+}\right)^{2}}{R_{2+}^{8}}+\eta_{2} \frac{\left(\mathbf{a}_{2} \cdot \mathbf{R}_{2+}\right)^{2}}{R_{2+}^{8}} \\
& +\eta_{3} \frac{1}{R_{2+}^{10}}\left[3\left(\mathbf{a}_{1} \cdot \mathbf{R}_{2+}\right)\left(\mathbf{a}_{2} \cdot \mathbf{R}_{2+}\right)-R_{2+}^{2}\left(\mathbf{a}_{1} \cdot \mathbf{a}_{2}\right)\right],
\end{aligned}
$$

where $\mathbf{R}_{2+}=\mathbf{R}+\nu \mathbf{a}_{2}$ is the distance between the dipole and the polarizable core of the neighboring molecule, and where the coefficients $\eta_{1,2,3}$ depend on the components of the molecular dipole and the molecular polarizability

$$
\begin{gathered}
\eta_{1}=\frac{3}{2} d_{\perp}^{2}\left(\alpha_{\|}-\alpha_{\perp}\right), \\
\eta_{2}=3\left(d_{\|}^{2}-\frac{1}{2} d_{\perp}^{2}\right) \alpha_{\perp},
\end{gathered}
$$




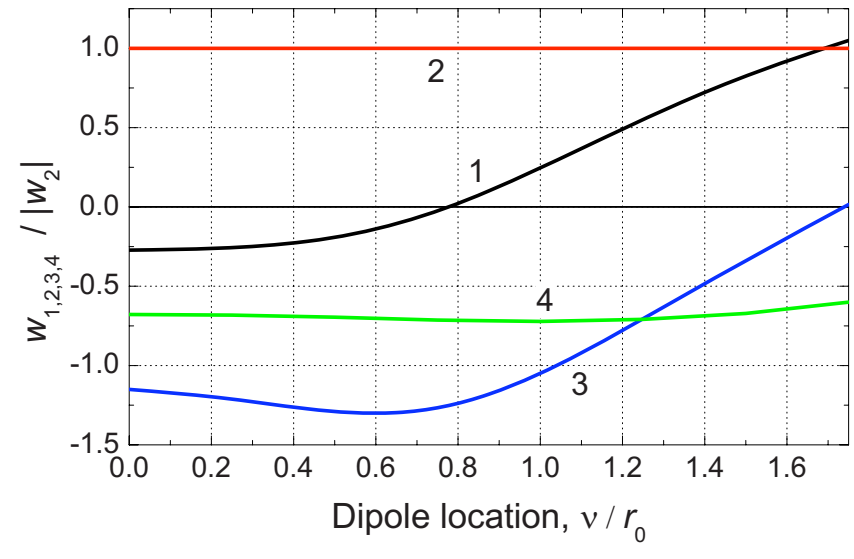

FIG. 14. (Color online) Normalized coefficients $w_{i} /\left|w_{2}\right|$ arising from the dipole-dipole induction interaction potential as a function of the dipole location $\nu$ calculated for the isotropic molecular polarizability and the hard rod cutoff $\kappa=4$.

$$
\eta_{3}=\left(d_{\|}^{2}-\frac{1}{2} d_{\perp}^{2}\right)\left(\alpha_{\|}-\alpha_{\perp}\right) .
$$

One notes that $\eta_{1}$ and $\eta_{3}$ vanish if one neglects the anisotropy of molecular polarizability. The coefficients $w_{1}-w_{3}$ calculated numerically for this simple case are presented in Fig. 14. Negative values of the constant $w_{4}$ indicate that the dipole-dipole induction interaction can also promote the $\mathrm{Sm} A-\mathrm{Sm} C$ phase transition. We have found out that the addition of this interaction to the Gay-Berne repulsionattraction indeed leads to the pronounced transition.

\section{CONCLUSIONS}

We have presented a simple phenomenological model and a detailed molecular theory which enable one to describe the properties of the $\operatorname{Sm} A-\operatorname{Sm} C$ transition in smectic liquid crystals with both conventional and anomalously low layer contraction. The phenomenological model yields explicit analytical expressions for the smectic layer spacing in the smectic- $A$ and $-C$ phases which have been used to produce very good fits of the experimental data for materials of different molecular structure and exhibiting different degrees of layer contraction.

In contrast to the previous models, the present statistical theory is based on a complete set of orientational order parameters of the $\mathrm{SmC}$ phase. In the case of the $\mathrm{SmC}$ phase composed of uniaxial molecules, there are three independent order parameters $S_{k}, P_{k}$, and $V$ which characterize the orientational ordering with respect to the smectic layer normal, the biaxiality in the smectic plane and the molecular tilt, respectively. These order parameters are defined in the laboratory frame as explicit statistical averages of the corresponding microscopic quantities. Thus in this theory the $\mathrm{Sm} A$-Sm $C$ transition is described as an order-disorder phase transition. The conventional order parameters $S$ and $P$ defined in the director frame as well as the tilt angle $\Theta$ are expressed in terms of $S_{k}, P_{k}$ and $V$ via the exact formulas (22), (23). One notes that from the experimental point of view it is more convenient to measure the order parameters in the laboratory frame because this can be done directly without additional measurements of the tilt angle.

Both phenomenological and the molecular theory indicate that the $\mathrm{Sm} A-\mathrm{SmC}$ transition occurs when the nematic order parameter $S$ exceeds some critical value. Thus a certain degree of orientational disorder, still present in the system, is required for the transition to occur. The present statistical theory is based on the systematic expansion of the effective pair intermolecular interaction potential, which contains several different terms. The properties of different types of tilting transition are then determined by an interplay between these terms, which have different roles in the case of partial orientational disorder. In practice, the behavior of the system depends on the coupling coefficients, which effectively are the model parameters. Therefore, the general results obtained in this paper are independent of a particular choice of intermolecular interactions.

At the same time, we have developed a procedure which enables one to calculate numerically the corresponding coupling constants for a given intermolecular potential. We have made such calculations for the standard Gay-Berne potential and its combinations with the electrostatic dipole-dipole and induction dipole-dipole interactions. It has been shown that, as expected, the Gay-Berne potential alone does not promote the $\mathrm{SmC}$ phase while both electrostatic and induction dipoledipole interactions may be responsible for the $\mathrm{Sm} A-\mathrm{SmC}$ transition. These particular results are confirmed by recent computer simulations $[14,15]$. We note, however, that the simple interaction models should not be taken too seriously because of the complexity of real organic mesogenic molecules. In fact, there exist a number of other possible interactions which may produce the same effect. Further conclusions here will depend on a careful estimate of the corresponding contributions for particular materials and certain molecular structure.

In spite of its relative simplicity, the present molecularstatistical model enables one to reproduce a broad variety of different transition scenarios. Apart from the conventional tilting transition, the theory describes the anomalously weak layer contraction in the smectic- $C$ phase observed in some smectic liquid crystals which are also often called the de Vries-type materials. It should be stressed, however, that the results of this paper do not support the original model of de Vries. Indeed, the de Vries model is based on the assumption that the orientational distribution of long molecular axes in the smectic- $A$ phase of a conventional smectic material is qualitatively different from that in smectics with anomalously weak layer contraction. In conventional smectics $A$ the long molecular axes are distributed around the layer normal while in other materials the axes are approximately distributed on the cone making a fixed angle with the layer normal. Even if the cone model is considered as a limiting case, there is still an assumption that orientational distributions are substantially different in the two different types of smectics $A$. In our theory, however, the shape of the orientational distribution function in the smectic- $A$ phase is qualitatively the same in conventional smectics and smectics that tilt almost without layer contraction. Thus the present theory suggests an alternative explanation of the weak layer contraction in the smectic- $C$ phase. 
In this theory, there are two major factors determining the temperature variation of the layer spacing. On the one hand, the layer spacing is decreasing with the increasing tilt. On the other hand, the spacing increases with the increasing nematic order parameter. These two factors may compensate each other to some extent if the nematic order parameter is relatively low in the smectic- $C$ phase and thus possesses a significant temperature dependence. As a result the ideal conventional layer contraction, determined by the factor of $\cos \Theta$, may occur only in materials with high and saturated nematic order parameter. Finally it has been shown in this paper that for relatively weak nematic order and for a certain class of intermolecular interaction potentials the compensation may be nearly complete, and the tilt in the smectic- $C$ phase will be accompanied by almost no layer contraction. In particular, there exists a simple relationship between the two coupling constants in the model interaction potential which enables one to reproduce the anomalously weak layer contraction regardless of the values of the remaining parameters.

The present theory also enables one to reproduce a correlation between the rapid growth of the nematic order parameter below the $\mathrm{Sm} A-\mathrm{SmC}$ transition, anomalously weak layer contraction, and the experimentally observed anomaly of the heat capacity which may correspond to the first order transition. The underlying physical phenomenon here is a coupling between the nematic order parameter and the tilt which enhances the nematic order, expands the smectic layers, and affects the temperature dependence of the order parameters and related thermodynamic characteristics. It has been shown that the experimentally observed anomaly of the heat capacity in some smectic materials can qualitatively be explained using the model of a second order $\operatorname{Sm} A-\operatorname{Sm} C$ transition which is close to the tricritical point.

Recently a phenomenological model describing the anomalously weak layer contraction has also been proposed by Saunders et al. [49]. The model is based on a coupling between the tensor order parameter and the wave vector of the smectic structure which is rather similar to the one considered in Sec. II, although some assumptions are different. Nevertheless, the authors arrive at the same general conclusion that the weak layer contraction in the smectic- $C$ phase is not related to any molecular pretilt in the smectic- $A$ phase, but is determined by temperature variation of the nematic order parameter and by specific restrictions on the values of phenomenological parameters. On the other hand, the model [49] predicts a coexistence between two different smectic- $A$ phases and a nonmonotonous behavior of the birefringence in the Sm- $A$ phase which does not follow from our model. This may be related to the temperature variation of the smectic order parameter which is not taken into account in the present model, or to specific assumptions about the temperature variation of the nematic order parameter made in Ref. [49].

Finally, we note that in this paper we have considered the $\mathrm{Sm} A-\mathrm{Sm} C$ transition in a system of effectively uniaxial molecules. Thus we have neglected the biaxial ordering of short molecular axes which is always present to some extent in any smectic- $C$ liquid crystal. A more detailed study of the influence of molecular biaxiality on the transition will be published separately.

\section{ACKNOWLEDGMENTS}

The authors are grateful to P. Collings, C. C. Huang, H. Gleeson, V. Lorman, Yu. Panarin, T. J. Sluckin, and J. K. Vij for valuable discussions, and to EPSRC (UK) for funding.

\section{APPENDIX: ORDER PARAMETERS IN SMECTIC- $C$ PHASE}

It is well known since the work of Straley [50] that the tensor order parameter which describes the ordering of long molecular axis a can be expressed in the diagonal form as

$$
Q_{i j}=S\left(n_{i} n_{j}-\frac{1}{3} \delta_{i j}\right)+P\left(m_{i} m_{j}-h_{i} h_{j}\right),
$$

where

$$
S=\left\langle P_{2}(\cos \Gamma)\right\rangle, \quad P=\left\langle\sin ^{2} \Gamma \cos 2 \Phi\right\rangle .
$$

Here the director $\mathbf{n}$ is the primary axis of the tensor $\mathbf{Q}$ which corresponds to the maximum eigenvalue, $S$ is the conventional orientational (nematic) order parameter which characterizes the ordering of long axes along the director, and $P$ is the biaxial order parameter which describes the biaxial distribution of long molecular axis around the director. The angles $\Gamma$ and $\Phi$ are defined as the polar and azimuthal angles of the molecular axis $\mathbf{a}$ in the director frame $(\mathbf{n}, \mathbf{m}, \mathbf{h})$.

In the smectic- $C$ phase, the director $\mathbf{n}$ is inclined with respect to the layer normal $\mathbf{k}$, while another primary axis $\mathbf{h}$ of the tensor $\mathbf{Q}$ is parallel to the twofold symmetry axis in the direction perpendicular to the tilt plane. Thus in the diagonal frame the tensor order parameter $\mathbf{Q}$ is specified by three parameters: scalar order parameters $S$ and $P$ and the tilt angle $\Theta$ between the director and the smectic layer normal.

In some cases the representation of the tensor $\mathbf{Q}$ in the diagonal frame is not very convenient because the orientation of the director may not be known (for example, in computer simulations). At the same time, the orientation of the smectic layer normal $\mathbf{k}$ is usually known and the tensor $\mathbf{Q}$ can also be represented in the frame $(\mathbf{k}, \mathbf{c}, \mathbf{h})$ (see Fig. 3) based on the layer normal $\mathbf{k}$ :

$$
Q_{i j}=S_{k}\left(k_{i} k_{j}-\delta_{i j} / 3\right)+\frac{1}{2} P_{k}\left(c_{i} c_{j}-h_{i} h_{j}\right)+\frac{1}{2} C\left(k_{i} c_{j}+c_{i} k_{j}\right),
$$

where

$S_{k}=\left\langle P_{2}(\cos \gamma)\right\rangle, \quad P_{k}=\left\langle\sin ^{2} \gamma \cos 2 \varphi\right\rangle, \quad V=\langle\sin 2 \gamma \cos \varphi\rangle$.

and where the angles $\gamma$ and $\varphi$ are the polar and azimuthal angles of $\mathbf{a}$ in the $\mathbf{k}$ frame. Accordingly, the tensor $\mathbf{Q}$ here is specified by another set of three order parameters $S_{k}, P_{k}$, and $V$. The parameter $S_{k}$ describes the ordering of long molecular axes with respect to the layer normal $\mathbf{k}$, and the parameter $P_{k}$ is the measure of biaxiality in the smectic plane. One notes that in the smectic- $C$ phase the tensor $\mathbf{Q}$ is not diagonal in the $\mathbf{k}$ frame, and the corresponding off-diagonal element $V$ describes the tilt. In the smectic $A$ phase, $V=0$ because the 
director $\mathbf{n}=\mathbf{k}$ and the tensor $\mathbf{Q}$ must be diagonal.

It is important to note that in Eqs. (A4) all three order parameters are explicitly expressed as statistical averages while for the representation Eq. (A1) the tilt angle $\Theta$ has more of a geometrical meaning. The tensor $\mathbf{Q}$ in the form (A3) can be diagonalized and thus expressed in the form of Eq. (A1). As a result, one obtains the following rigorous relationships between the two sets of order parameters:

$$
\begin{gathered}
\tan 2 \Theta=\frac{V}{S_{k}-0.5 P_{k}}, \\
S=\frac{1}{4} S_{k}+\frac{3}{8} P_{k}+\frac{3 V}{4 \sin 2 \Theta},
\end{gathered}
$$

$$
P=\frac{1}{2} S_{k}+\frac{3}{4} P_{k}-\frac{V}{2 \sin 2 \Theta} .
$$

One can readily see that the tilt order parameter $V$ is a monotonously growing function of $\Theta$, and at small $\Theta, V \propto \Theta$.

The order parameters $P_{k}, S_{k}$ and $V$ are particularly useful when there exist several ordering second rank tensors which may correspond to different molecular fragments or to long and short molecular axes. All such tensors can be expressed in the diagonal form (A1). At the same time, the orientation of the director in the tilt plane of the smectic- $C$ phase is not specified by any symmetry, and thus in the general case different ordering tensors will correspond to different directors and different tilt angles. This confusion may be avoided using the order parameters $S_{k}, P_{k}$, and $V$ for every ordering tensor.
[1] J. P. F. Lagerwall and F. Giesselmann, ChemPhysChem 7, 20 (2006).

[2] G. Pelz, S. Diele, and W. Weissflog, Adv. Mater. (Weinheim, Ger.) 11, 707 (1999).

[3] S. Lagerwall, Ferroelectric and Antiferroelectric Liquid Crystals (Wiley-VCH, Weinheim, 1999).

[4] W. Lehmann, H. Skupin, C. Tolksdorf, E. Gebhard, R. Zentel, P. Krüger, M. Lösche, and F. Kremer, Nature (London) 410, 447 (2001).

[5] O. Stenull and T. C. Lubensky, Phys. Rev. Lett. 94, 018304 (2005).

[6] V. M. Kaganer, H. Möwald, and P. Dutta, Rev. Mod. Phys. 71, 779 (1999).

[7] T. R. Taylor, S. L. Arora, and J. L. Fergason, Phys. Rev. Lett. 25, 722 (1970).

[8] W. L. McMillan, Phys. Rev. A 4, 1238 (1971).

[9] A. Wulf, Phys. Rev. A 11, 365 (1975).

[10] A. M. Somoza and P. Tarazona, Phys. Rev. Lett. 61, 2566 (1988).

[11] B. W. van der Meer and G. Vertogen, J. Phys. (Paris), Colloq. 40, C3-222 (1979).

[12] W. J. A. Goossens, J. Phys. (Paris) 46, 1411 (1985).

[13] A. Poniwierski and T. J. Sluckin, Mol. Phys. 73, 199 (1991).

[14] M. P. Neal and A. J. Parker, Chem. Phys. Lett. 294, 277 (1998).

[15] R. Berardi, S. Orlandi, and C. Zannoni, Phys. Rev. E 67, 041708 (2003).

[16] A. S. Govind and N. V. Madhusudana, Europhys. Lett. 55, 505 (2001).

[17] P. G. de Gennes and J. Prost, The Physics of Liquid Crystals (Clarendon, Oxford, 1993).

[18] L. A. Beresnev, L. M. Blinov, M. A. Osipov, and S. A. Pikin, Mol. Cryst. Liq. Cryst. 158, 1 (1988).

[19] T. P. Rieker, N. A. Clark, G. S. Smith, D. S. Parmar, E. B. Sirota, and C. R. Safinya, Phys. Rev. Lett. 59, 2658 (1987).

[20] Y. Takanishi, Y. Ouchi, H. Takezoe, A. Fukuda, A. Mochizuki, and M. Nakatsuka, Jpn. J. Appl. Phys., Part 2 29, L984 (1990)

[21] J. Naciri, J. Ruth, G. Crawford, R. Shashidhar, and B. R. Ratna, Chem. Mater. 7, 1397 (1995).
[22] M. D. Radcliffe, M. L. Brostrom, K. A. Epstein, A. G. Rappaport, B. N. Thomas, R. F. Shao, and N. A. Clark, Liq. Cryst. 26, 789 (1999).

[23] F. Giesselmann, P. Zugenmaier, I. Dierking, S. T. Lagerwall, B. Stebler, M. Kaspar, V. Hamplova, and M. Glogarova, Phys. Rev. E 60, 598 (1999).

[24] E. Velasco, L. Mederos, and T. J. Sluckin, Liq. Cryst. 20, 399 (1996).

[25] A. de Vries, Mol. Cryst. Liq. Cryst. 11, 361 (1970).

[26] A. de Vries, J. Chem. Phys. 71, 25 (1979).

[27] A. de Vries, Mol. Cryst. Liq. Cryst. 41, 27 (1977).

[28] A. de Vries, A. Ekachai, and N. Spielberg, Mol. Cryst. Liq. Cryst. 49, 143 (1979).

[29] A. de Vries, in Advances in Liquid Crystal Research and Applications, edited by L. Bata (Pergamon Press, Oxford, 1980).

[30] A. J. Leadbetter and E. K. Norris, Mol. Phys. 38, 669 (1979).

[31] J. P. F. Lagerwall, F. Giesselmann, and M. D. Radcliffe, Phys. Rev. E 66, 031703 (2002).

[32] J. V. Selinger, P. J. Collings, and R. Shashidhar, Phys. Rev. E 64, 061705 (2001).

[33] R. Korlacki, A. Fukuda, and J. K. Vij, Europhys. Lett. 77, 36004 (2007).

[34] C. C. Huang, S. T. Wang, X. F. Han, A. Cady, R. Pindak, W. Caliebe, K. Ema, K. Takekoshi, and H. Yao, Phys. Rev. E 69, 041702 (2004)

[35] M. V. Gorkunov, F. Giesselmann, J. P. F. Lagerwall, T. J. Sluckin, and M. A. Osipov, Phys. Rev. E 75, 060701(R) (2007).

[36] J.-h. Chen and T. C. Lubensky, Phys. Rev. A 14, 1202 (1976).

[37] P. K. Mukherjee, H. Pleiner, and H. R. Brand, J. Chem. Phys. 117, 7788 (2002).

[38] P. Bescari (private communication).

[39] Y. Singh, Phys. Rev. A 30, 583 (1984).

[40] T. J. Sluckin and P. Shukla, J. Phys. A 16, 1539 (1983).

[41] M. A. Osipov, in Handbook of Liquid Crystals, 2nd ed., edited by D. Demus, J. Goodby, G. W. Gray, H.-W. Spies, and V. Vill (Wiley-VCH, Weinheim, 1998), Vol. 1.

[42] N. Hayashi, A. Kocot, M. J. Linehan, A. Fukuda, J. K. Vij, G. Heppke, J. Naciri, S. Kawada, and S. Kondoh, Phys. Rev. E 
74, 051706 (2006).

[43] K. Merkel, A. Kocot, J. K. Vij, G. H. Mehl, and T. Meyer, J. Chem. Phys. 121, 5012 (2004).

[44] M. D. Ossowska-Chrusciel, R. Korlacki, A. Kocot, R. Wrzalik, J. Chrusciel, and S. Zalewski, Phys. Rev. E 70, 041705 (2004).

[45] F. Giesselmann and P. Zugenmaier, Phys. Rev. E 55, 5613 (1997).

[46] J. G. Gay and B. J. Berne, J. Chem. Phys. 74, 3316 (1981).
[47] E. de Miguel, L. F. Rull, M. K. Chalam, K. E. Gubbins, and F. Van Swol, Mol. Phys. 72, 593 (1991).

[48] R. Berardi, A. J. Emerson, and C. Zannoni, J. Chem. Soc., Faraday Trans. 89, 4069 (1993).

[49] K. Saunders, D. Hernandez, S. Pearson, and John Toner, Phys. Rev. Lett. 98, 197801 (2007).

[50] J. P. Straley, Phys. Rev. A 10, 1881 (1974). 